\title{
On the Uniqueness of Hofer's Geometry
}

\author{
Lev Buhovsky, Yaron Ostrover
}

November 8, 2018

\begin{abstract}
We study the class of norms on the space of smooth functions on a closed symplectic manifold, which are invariant under the action of the group of Hamiltonian diffeomorphisms. Our main result shows that any such norm that is continuous with respect to the $C^{\infty}$-topology, is dominated from above by the $L_{\infty}$-norm. As a corollary, we obtain that any bi-invariant Finsler pseudo-metric on the group of Hamiltonian diffeomorphisms that is generated by an invariant norm that satisfies the aforementioned continuity assumption, is either identically zero or equivalent to Hofer's metric.
\end{abstract}

\section{Introduction and Main Results}

A remarkable fact, which is among the cornerstones of symplectic rigidity theory, is that the group of Hamiltonian diffeomorphisms of a symplectic manifold can be equipped with an intrinsic geometry given by a bi-invariant Finsler metric known as Hofer's metric. In contrast with finite-dimensional Lie groups, the existence of such a metric on an infinite-dimensional group of transformations is highly unusual due to the lack of compactness. In the past twenty years, Hofer's metric has been intensively studied with many new discoveries covering a wide range of aspects in Hamiltonian dynamics and symplectic geometry.

The purpose of this note is to show that under some mild assumption, Hofer's metric is, in a sense, the only bi-invariant Finsler metric on the group of Hamiltonian diffeomorphisms of closed symplectic manifolds. In order to state this result precisely we proceed with some standard definitions and notations, and refer the reader to the books [7, 11, 15] for symplectic preliminaries, and further discussions on the group of Hamiltonian diffeomorphisms and Hofer's geometry.

Let $(M, \omega)$ be a closed $2 n$-dimensional symplectic manifold, and denote by $C_{0}^{\infty}(M)$ the space of smooth functions that are zero-mean normalized with respect to the 
canonical volume form $\omega^{n}$. For every smooth time-dependent Hamiltonian function $H: M \times[0,1] \rightarrow \mathbb{R}$, we associate a vector field $X_{H_{t}}$ via the equation $i_{X_{H_{t}}} \omega=-d H_{t}$, where $H_{t}(x)=H(t, x)$. The flow of $X_{H_{t}}$ is denoted by $\phi_{H}^{t}$ and is defined for all $t \in[0,1]$. The main object of this note is the group of Hamiltonian diffeomorphisms, which consists of all the time-one maps of such Hamiltonian flows i.e.,

$$
\operatorname{Ham}(M, \omega)=\left\{\phi_{H}^{1} \mid \text { where } \phi_{H}^{t} \text { is a Hamiltonian flow }\right\}
$$

When equipped with the standard $C^{\infty}$-topology, the group $\operatorname{Ham}(M, \omega)$ is an infinitedimensional Fréchet Lie group, whose Lie algebra $\mathcal{A}$ can be identified with the space $C_{0}^{\infty}(M)$. Moreover, the adjoint action of $\operatorname{Ham}(M, \omega)$ on $\mathcal{A}$ is the standard action of diffeomorphisms on functions i.e., $A d_{\phi} f=f \circ \phi^{-1}$, for every $f \in \mathcal{A}$ and $\phi \in$ $\operatorname{Ham}(M, \omega)$. Next, we define a Finsler (pseudo) distance on $\operatorname{Ham}(M, \omega)$. Given any norm $\|\cdot\|$ on the Lie algebra $\mathcal{A}$, we define the length of a path $\alpha:[0,1] \rightarrow \operatorname{Ham}(M, \omega)$ as

$$
\text { length }\{\alpha\}=\int_{0}^{1}\|\dot{\alpha}\| d t=\int_{0}^{1}\left\|H_{t}\right\| d t,
$$

where $H_{t}(x)=H(t, x)$ is the unique normalized Hamiltonian function generating the path $\alpha$. Here $H$ is said to be normalized if $\int_{M} H_{t} \omega^{n}=0$ for every $t \in[0,1]$. The distance between two Hamiltonian diffeomorphisms is given by

$$
d(\psi, \varphi):=\inf \text { length }\{\alpha\},
$$

where the infimum is taken over all Hamiltonian paths $\alpha$ connecting $\psi$ and $\varphi$. It is not hard to check that $d$ is non-negative, symmetric and satisfies the triangle inequality. Moreover, a norm on $\mathcal{A}$ which is invariant under the adjoint action yields a bi-invariant pseudo-distance function, i.e. $d(\psi, \phi)=d(\theta \psi, \theta \phi)=d(\psi \theta, \phi \theta)$ for every $\psi, \phi, \theta \in \operatorname{Ham}(M, \omega)$. From now on we will deal solely with such norms 1 and we will refer to $d$ as the pseudo-distance generated by the norm $\|\cdot\|$.

Remark 1.1. When one studies the geometric properties of the group of Hamiltonian diffeomorphisms, it is convenient to consider smooth paths $[0,1] \rightarrow \operatorname{Ham}(M, \omega)$, among which, those that start at the identity correspond to smooth Hamiltonian flows. Moreover, for a given Finsler metric on $\operatorname{Ham}(M, \omega)$, a natural assumption from a geometric point of view is that every smooth path $[0,1] \rightarrow \operatorname{Ham}(M, \omega)$ is of a finite length. As it turns out, the latter assumption is equivalent to the continuity of the norm on $\mathcal{A}$ corresponding to the Finsler metric in the $C^{\infty}$-topology 2 . We prove

\footnotetext{
${ }^{1}$ We remark that a fruitful study of right-invariant Finsler metrics on $\operatorname{Ham}(M, \omega)$, motivated in part by applications to hydrodynamics, was initiated in a well known paper by Arnold 1] (see also [2], 8] and the references within). Moreover, non-Finslerian bi-invariant metrics on $\operatorname{Ham}(M, \omega)$ have been intensively studied in the realm of symplectic geometry, starting with the works of Viterbo [18, Schwarz [17, and Oh [12, and followed by many others.

${ }^{2}$ We thank A. Katok for his illuminating remark regarding the naturalness of the assumption that the norm is continuous in the $C^{\infty}$-topology.
} 
this fact in the Appendix to the paper. Throughout the text we shall consider only such norms.

It is highly non-trivial to check whether a distance function generated by such a norm, is non-degenerate, that is $d(\mathbb{1}, \phi)>0$ for $\phi \neq 1$. In fact, for closed symplectic manifolds, a bi-invariant pseudo-metric $d$ on $\operatorname{Ham}(M, \omega)$ is either a genuine metric or identically zero. This is an immediate corollary of a well known theorem by Banyaga [3], which states that $\operatorname{Ham}(M, \omega)$ is a simple group, combined with the fact that the null-set

$$
\operatorname{null}(d)=\{\phi \in \operatorname{Ham}(M, \omega) \mid d(\mathbb{1}, \phi)=0\}
$$

is a normal subgroup of $\operatorname{Ham}(M, \omega)$. A distinguished result by Hofer [6] states that the $L_{\infty}$-norm on $\mathcal{A}$ gives rise to a genuine distance function on $\operatorname{Ham}(M, \omega)$ known as Hofer's metric. This was discovered and proved by Hofer for the case of $\mathbb{R}^{2 n}$, then generalized by Polterovich [14], and finally proven in full generality by Lalonde and McDuff [10]. In a sharp contrast to the above, Eliashberg and Polterovich [5] showed that for $1 \leq p<\infty$, the pseudo-distances on $\operatorname{Ham}(M, \omega)$ corresponding to the $L_{p^{-}}$ norms on $\mathcal{A}$ vanishes identically. A considerable generalization of the latter result was given by Ostrover-Wagner [13] who proved that for a closed symplectic manifold:

Theorem 1.2 (Ostrover-Wagner [13]). Let $\|\cdot\|$ be a $\operatorname{Ham}(M, \omega)$-invariant norm on $\mathcal{A}$ such that $\|\cdot\| \leq C\|\cdot\|_{\infty}$ for some constant $C$, but the two norms are not equivalent. Then the associated pseudo-distance d on $\operatorname{Ham}(M, \omega)$ vanishes identically.

In [5], the authors started a discussion regarding the uniqueness of Hofer's metric (cf. [4], [15]). For the case of closed symplectic manifolds, one question they arose is:

Question: Does there exist a Finsler bi-invariant metric on $\operatorname{Ham}(M, \omega)$ which is not equivalent to Hofer's metric.

In this paper we provide an answer to the above question under the natural continuity assumption mentioned in Remark 1.1. More precisely, our main result is:

Theorem 1.3. Let $(M, \omega)$ be a closed symplectic manifold. Any $\operatorname{Ham}(M, \omega)$-invariant pseudo norm $\|\cdot\|$ on $\mathcal{A}$ that is continuous in the $C^{\infty}$-topology, is dominated from above by the $L_{\infty}$-norm i.e., $\|\cdot\| \leq C\|\cdot\|_{\infty}$ for some constant $C$.

Combining together Theorem 1.3 and Theorem 1.2, we conclude that:

Corollary 1.4. For a closed symplectic manifold $(M, \omega)$, any bi-invariant Finsler pseudo metric on $\operatorname{Ham}(M, \omega)$, obtained by a pseudo norm $\|\cdot\|$ on $\mathcal{A}$ that is continuous in the $C^{\infty}$-topology, is either identically zero or equivalen 3 to Hofer's metric. In

\footnotetext{
${ }^{3}$ Here two metrics $d_{1}, d_{2}$ are said to be equivalent if $\frac{1}{C} d_{1} \leqslant d_{2} \leqslant C d_{1}$ for some constant $C>0$.
} 
particular, any non-degenerate bi-invariant Finsler metric on $\operatorname{Ham}(M, \omega)$, which is generated by a norm that is continuous in the $C^{\infty}$-topology, gives rise to the same topology on $\operatorname{Ham}(M, \omega)$ as the one induced by Hofer's metric.

Remark 1.5. Let us emphasize that any norm $\|\cdot\|$ on $\mathcal{A}$ can be turned into a $\operatorname{Ham}(M, \omega)$-invariant pseudo-norm via the invariantization procedure $\|f\| \mapsto\|f\|_{i n v}$, where:

$$
\|f\|_{i n v}=\inf \left\{\sum\left\|\phi_{i}^{*} f_{i}\right\| ; f=\sum f_{i}, \text { and } \phi_{i} \in \operatorname{Ham}(M, \omega)\right\}
$$

Note that $\|\cdot\|_{i n v} \leq\|\cdot\|$. Thus, if $\|\cdot\|$ is continuous in the $C^{\infty}$-topology, then so is $\|\cdot\|_{i n v}$. Moreover if $\|\cdot\|^{\prime}$ is a $\operatorname{Ham}(M, \omega)$-invariant norm, then:

$$
\|\cdot\|^{\prime} \leq\|\cdot\| \Longrightarrow\|\cdot\|^{\prime} \leq\|\cdot\|_{i n v}
$$

In particular, the above invariantization procedure provides a plethora of $\operatorname{Ham}(M, \omega)$ invariant genuine norms on $\mathcal{A}$, e.g., by taking the homogenization of the $\|\cdot\|_{C^{k}}$-norms.

Structure of the paper: In Section 2 we sketch an outline of the proof of Theorem 1.3. In Section 3 we prove a local version of this theorem, which would serve as the main ingredient in the proof of the general case given in Section 4 .

Notations: Let $x_{1}, \ldots, x_{n}$ be the Cartesian coordinates in $\mathbb{R}^{n}$. For any multi-index $\alpha=\left(\alpha_{1}, \ldots, \alpha_{n}\right)$, set $\partial^{\alpha}=\partial_{1}^{\alpha_{1}} \partial_{2}^{\alpha_{2}} \ldots \partial_{n}^{\alpha_{n}}$, where $\partial_{i}=\partial / \partial x_{i}$. For an open set $\Omega \subset \mathbb{R}^{n}$ we denote $C_{c}(\Omega)$ the space of compactly supported continuous functions on $\Omega$, and let $\|\cdot\|_{\infty}$ stands for the $L_{\infty}$-norm. For an integer $k$, define $C_{c}^{k}(\Omega)$ the class of functions $f$ from $C_{c}(\Omega)$ such that $\partial^{\alpha} f \in C_{c}(\Omega)$ for all $|\alpha| \leq k$. The $C^{k}$-norm of $u \in C_{c}^{k}(\Omega)$ is given by

$$
\|u\|_{C^{k}}=\max _{|\alpha| \leq k} \sup _{\Omega}\left|\partial^{\alpha} u\right|
$$

As usual, $C_{c}^{\infty}(\Omega)$ is the intersection of all the $C_{c}^{k}(\Omega)$ and is endowed with the $C^{\infty}$ topology. We denote by $\operatorname{supp}(f)$ the support of the function $f$ i.e., the closure of the set $\{x \mid f(x) \neq 0\}$, and by $\operatorname{int}(D)$ the interior of a domain $D \subset \mathbb{R}^{n}$. For an open domain $U \subset \mathbb{R}^{2 n}$, we denote by $\operatorname{Ham}_{c}(D, \omega)$ the group of Hamiltonian diffeomorphisms of $\mathbb{R}^{2 n}$, which are generated by Hamiltonian functions $H: \mathbb{R}^{2 n} \times[0,1] \rightarrow \mathbb{R}$, whose support is compact and contained in $U \times[0,1]$. Here $\omega$ is the standard symplectic form on $\mathbb{R}^{2 n}$ given by $\omega=d p \wedge d q$, where $\left\{q_{1}, p_{1}, \ldots, q_{n}, p_{n}\right\}$ are the canonical coordinates in $\mathbb{R}^{2 n}$. We say that a function $f: \mathbb{R}^{2 n} \rightarrow \mathbb{R}$ is a product function, if it is of the form $f(q, p)=\prod_{i=1}^{n} f_{i}\left(q_{i}, p_{i}\right)$. Finally, the letters $C, C_{1}, C_{2}, \ldots$ are used to denote positive constants that depend solely on the dimension of the ambient space relevant in each particular context.

Acknowledgements: Both authors are grateful to H. Hofer and L. Polterovich, for their interest in this work and helpful comments. This article was written during visits of the first author at the Institute for Advanced Study (IAS) in Princeton, and 
visits of the second author at the Mathematical Sciences Research Institute (MSRI), Berkeley. We thank these institutions for their stimulating working atmospheres and for financial support. The first author was supported by the Mathematical Sciences Research Institute. The second author was supported by NSF Grant DMS-0635607, and by the Israel Science Foundation grant No. 1057/10. Any opinions, findings and conclusions or recommendations expressed in this material are those of the authors and do not necessarily reflect the views of the NSF or the ISF.

\section{Outline of the Proof}

Here we briefly describe the strategy of the proof of Theorem 1.3. For technical reasons, we shall prove Theorem 1.3 for norms on the space $C^{\infty}(M)$, instead of the space $\mathcal{A}$. The original claim would follow from this result since any $\operatorname{Ham}(M, \omega)$ invariant pseudo-norm $\|\cdot\|$ on $\mathcal{A}$ can be naturally extended to an invariant pseudonorm $\|\cdot\|^{\prime}$ on $C^{\infty}(M)$ by setting

$$
\|f\|^{\prime}=\left\|f-M_{f}\right\|, \text { where } M_{f}=\frac{1}{\operatorname{Vol}(M)} \int_{M} f \omega^{n}
$$

Note that if $\|\cdot\|$ is continuous in the $C^{\infty}$-topology, then so is $\|\cdot\|^{\prime}$. Moreover, the norm $\|\cdot\|^{\prime}$ coincides with $\|\cdot\|$ on the space $\mathcal{A}$. By a standard partition of unity argument, we reduce the proof of the theorem to a "local result", i.e., we show that it is sufficient to prove Theorem 1.3 for $\operatorname{Ham}_{c}(W, \omega)$-invariant norms on $C_{c}^{\infty}(W)$, where $W=(-L, L)^{2 n}$ is a $2 n$-dimensional cube in $\mathbb{R}^{2 n}$. As a first step toward this end, we introduce a special $\operatorname{Ham}_{c}(W, \omega)$-invariant norm $\|\cdot\|_{\mathcal{F}, \text { max }}$ on $C_{c}^{\infty}(W)$, which depends on a given finite collection $\mathcal{F} \subset C_{c}^{\infty}(W)$. More precisely:

Definition I. For a non-empty finite collection $\mathcal{F} \subset C_{c}^{\infty}(W)$, let

$$
\mathcal{L}_{\mathcal{F}}:=\left\{\sum_{i} c_{i} \Phi_{i}^{*} f_{i} \mid c_{i} \in \mathbb{R}, \Phi_{i} \in \operatorname{Ham}_{c}(W, \omega), f_{i} \in \mathcal{F}, \text { and } \#\left\{i \mid c_{i} \neq 0\right\}<\infty\right\},
$$

be equipped with the norm

$$
\|f\|_{\mathcal{L}_{\mathcal{F}}}=\inf \sum\left|c_{i}\right|
$$

where the infimum is taken over all the representations $f=\sum c_{i} \Phi_{i}^{*} f_{i}$ as above.

Definition II. For any compactly supported function $f \in C_{c}^{\infty}(W)$, let

$$
\|f\|_{\mathcal{F}, \max }=\inf \left\{\liminf _{i \rightarrow \infty}\left\|f_{i}\right\|_{\mathcal{L}_{\mathcal{F}}}\right\}
$$

where the infimum is taken over all subsequences $\left\{f_{i}\right\}$ in $\mathcal{L}_{\mathcal{F}}$ which converge to $f$ in the $C^{\infty}$-topology. As usual, the infimum of the empty set is set to be $+\infty$. 
The main feature of the norm $\|\cdot\|_{\mathcal{F}, \max }$ is that it dominates from above any other $\operatorname{Ham}_{c}(W, \omega)$-invariant norm that is continuous in the $C^{\infty}$-topology (see Lemma 3.3). The next step, which is also the main part of the proof, is to show that for a suitable collection of functions $\mathcal{F} \subset C_{c}^{\infty}(W)$, the norm $\|\cdot\|_{\mathcal{F}, \text { max }}$ is in turn dominated from above by the $L_{\infty}$-norm. This is proved in Theorem 3.4, and in light of the above, it completes the proof of Theorem 1.3 . The proof of Theorem 3.4 is divided into two main steps which we now turn to describe:

The local two-dimensional case: Here, we shall construct a collection $\mathcal{F}$ of smooth compactly supported functions on a two-dimensional cube $W^{2} \subset \mathbb{R}^{2 n}$, such that any $f \in C_{c}^{\infty}\left(W^{2}\right)$ satisfies $\|f\|_{\mathcal{F}, \max } \leqslant C\|f\|_{\infty}$ for some absolute constant $C$. There are two independent components in the proof of this claim. First, we show that one can decompose any $f \in C_{c}^{\infty}\left(W^{2}\right)$ with $\|f\|_{\infty} \leqslant 1$ into a finite combination $f=\sum_{i=1}^{N_{0}} \epsilon_{j} \Psi_{j}^{*} g_{j}$. Here, $\epsilon_{j} \in\{-1,1\}, \Psi_{j} \in \operatorname{Ham}_{c}\left(W^{2}, \omega\right)$, and $g_{j}$ are smooth radial functions whose $L_{\infty}$-norm is bounded by an absolute constant, and which satisfy certain other technical conditions (see Proposition 3.5 for the precise statement). In what follows we call such functions by "simple functions". We emphasize that $N_{0}$ is a constant independent of $f$. Thus, we can restrict ourselves to the case where $f$ is a "simple function". In the second part of the proof, we construct an explicit collection $\mathcal{F}=\left\{\mathfrak{f}_{0}, \mathfrak{f}_{1}, \mathfrak{f}_{2}\right\}$, where $\mathfrak{f}_{i} \in C_{c}^{\infty}\left(W^{2}\right)$, and $i=0,1,2$. Using an averaging procedure (Proposition [3.6), we show that every "simple function" $f \in C_{c}^{\infty}\left(W^{2}\right)$ can be approximated arbitrarily well in the $C^{\infty}$-topology by a sum of the form

$$
\sum_{i, k} \alpha_{i, k} \widetilde{\Psi}_{i, k}^{*} \mathfrak{f}_{k}, \text { where } \widetilde{\Psi}_{i, k} \in \operatorname{Ham}_{c}\left(W^{2}, \omega\right), k \in\{0,1,2\}
$$

and such that $\sum\left|\alpha_{i, k}\right| \leq C\|f\|_{\infty}$ for some absolute constant $C$. Combining this with the above definiton of $\|\cdot\|_{\mathcal{F}, \max }$, we conclude that $\|f\|_{\mathcal{F}, \max } \leq C\|f\|_{\infty}$, for every $f \in C_{c}^{\infty}\left(W^{2}\right)$. This completes the proof of Theorem 3.4 in the 2-dimensional case.

The local higher-dimensional case: The proof of Theorem 3.4 for arbitrary dimension strongly relies on the 2-dimensional case. We extend (in a natural way) the construction of the above mentioned collection $\mathcal{F}=\left\{\mathfrak{f}_{0}, \mathfrak{f}_{1}, \mathfrak{f}_{2}\right\}$ to the $2 n$-dimensional case. By abuse of notation, we shall denote the new collection by $\mathcal{F}$ as well. Based on the proof of Theorem 3.4 in the 2-dimensional case, and on the construction of the class $\mathcal{F}$, we show that Theorem 3.4 holds for "product functions", i.e., for $f \in C_{c}^{\infty}(W)$ of the form $f=\prod_{i=1}^{n} f_{i}\left(q_{i}, p_{i}\right)$, where $f_{i} \in C_{c}^{\infty}\left(W^{2}\right)$. From this we derive, using a Fourier series argument, that the norm $\|\cdot\|_{\mathcal{F}, \max }$ is dominated from above by the $\|\cdot\|_{C^{2 n+1}}$-norm, i.e., for any $f \in C_{c}^{\infty}(W)$ one has

$$
\|f\|_{\mathcal{F}, \max } \leq C\|f\|_{C^{2 n+1}}
$$

for some constant $C$ (see Proposition 3.14 for the proof of the above two claims). Next, for any $\epsilon>0$, we construct a partition of unity function $\mathcal{R}^{\epsilon}: \mathbb{R}^{2 n} \rightarrow \mathbb{R}$, with 
$\operatorname{supp}\left(\mathcal{R}^{\epsilon}\right) \subset(-\epsilon, \epsilon)^{2 n}$, and such that

$$
\sum_{v \in \epsilon \mathbb{Z}^{2 n}} \mathcal{R}^{\epsilon}(x-v)=\mathbb{1}(x)
$$

For any $w \in \mathfrak{X}:=\{0,1,2,3\}^{2 n}$, we consider a finite grid $\Gamma_{w}^{\epsilon} \subset W$ given by:

$$
\Gamma_{w}^{\epsilon}=\epsilon w+4 \epsilon \mathbb{Z}^{2 n} \cap(-L+3 \epsilon, L-3 \epsilon)^{2 n},
$$

and define

$$
f_{w}(x)=\sum_{v \in \Gamma_{w}^{\epsilon}} \mathcal{R}^{\epsilon}(x-v) f(x)
$$

Note, that for $\epsilon$ sufficiently small such that $\operatorname{supp}(f) \subset(-L+4 \epsilon, L-4 \epsilon)^{2 n}$, one has

$$
f(x)=\sum_{w \in \mathfrak{X}} f_{w}(x)
$$

For any $w \in \mathfrak{X}$, the function $f_{w}$ is a finite sum of smooth functions that lie near the points of the grid $\Gamma_{w}^{\epsilon}$. Moreover, these functions have mutually disjoint supports, which are spaced commodiously. Next, we fix $w \in \mathfrak{X}$, and for any $v \in \Gamma_{w}^{\epsilon}$ we consider the decomposition of $f \in C_{c}^{\infty}(W)$ as a Taylor polynomial of order $2 n+1$ and a remainder, around the point $v$ (this specific choice of the order ensure, based on (2.1), the estimate (2.2) below):

$$
f(x)=P_{2 n+1}^{v}(x-v)+R_{2 n+1}^{v}(x-v) .
$$

We decompose each $f_{w}$ as $f_{w}(x)=g_{w}(x)+h_{w}(x)$, where

$$
g_{w}(x)=\sum_{v \in \Gamma_{w}^{\epsilon}} \mathcal{R}^{\epsilon}(x-v) P_{2 n+1}^{v}(x-v), \text { and } h_{w}(x)=\sum_{v \in \Gamma_{w}^{\epsilon}} \mathcal{R}^{\epsilon}(x-v) R_{2 n+1}^{v}(x-v) .
$$

Based on (2.1), in Lemma 3.16 (cf. Corrolary 3.17) we show that the $\|\cdot\|_{\mathcal{F}, \text { max }}$-norm of the reminder parts $\left\{h_{w}\right\}$ can be taken to be arbitrarily small. More precisely,

$$
\left\|h_{w}\right\|_{\mathcal{F}, \max } \leqslant C_{1}\left\|h_{w}\right\|_{C^{2 n+1}} \leqslant C_{2} \epsilon\|f\|_{C^{2 n+2}}
$$

for some constants $C_{1}$ and $C_{2}$. On the other hand, using a combinatorial argument and the above mentioned fact that Theorem 3.4 holds for "product functions", we prove the estimate

$$
\left\|g_{w}\right\|_{\mathcal{F}, \max } \leqslant C_{3}\left(\sum_{i=0}^{2 n+1}\|f\|_{C^{i}} \epsilon^{i}\right)
$$

for some constnat $C_{3}$. Combining the above estimates $(2.2)$ and $(2.3)$ for all $w \in \mathfrak{X}$, and taking $\epsilon \rightarrow 0$, we conclude that for every $f \in C_{c}^{\infty}(W)$ one has

$$
\|f\|_{\mathcal{F}, \max } \leqslant C_{4}\|f\|_{\infty}
$$

for some absolute constant $C_{4}$. This completes the proof of the theorem. 


\section{A Local Version of the Main Result}

In this section we prove a local version of our main result (Theorem 3.4 below), which would later serve as the main component in the proof of Theorem 1.3.

Consider an open cube $W=I^{2 n} \subset \mathbb{R}^{2 n}$, where $I=(-L, L) \subset \mathbb{R}$ is an open interval. Endow $W$ with linear coordinates $\left(q_{1}, p_{1}, \ldots, q_{n}, p_{n}\right)$, and with the standard symplectic structure $\omega=d p \wedge d q$ descending from $\mathbb{R}^{2 n}$. For a finite non-empty collection $\mathcal{F}$ of functions in $C_{c}^{\infty}(W)$, we define the space

$$
\mathcal{L}_{\mathcal{F}}:=\left\{\sum_{i} c_{i} \Phi_{i}^{*} f_{i} \mid c_{i} \in \mathbb{R}, \Phi_{i} \in \operatorname{Ham}_{c}(W, \omega), f_{i} \in \mathcal{F}, \text { and } \#\left\{i \mid c_{i} \neq 0\right\}<\infty\right\}
$$

We equip $\mathcal{L}_{\mathcal{F}}$ with the norm

$$
\|f\|_{\mathcal{L}_{\mathcal{F}}}:=\inf \sum\left|c_{i}\right|
$$

where the infimum is taken over all the representations $f=\sum c_{i} \Phi_{i}^{*} f_{i}$ as above.

Definition 3.1. For any compactly supported function $f \in C_{c}^{\infty}(W)$, let

$$
\|f\|_{\mathcal{F}, \max }=\inf \left\{\liminf _{i \rightarrow \infty}\left\|f_{i}\right\|_{\mathcal{L}_{\mathcal{F}}}\right\}
$$

where the infimum is taken over all subsequences $\left\{f_{i}\right\}$ in $\mathcal{L}_{\mathcal{F}}$ which converge to $f$ in the $C^{\infty}$-topology. If such sequence do not exists, we set $\|f\|_{\mathcal{F}, \max } \equiv+\infty$.

Remark 3.2. It follows from the definition above that $\left\|_{\cdot} \cdot\right\|_{\mathcal{F}, \max }$ is homogeneous, $\operatorname{Ham}_{c}(W, \omega)$-invariant, and satisfies the triangle inequality 4 . Moreover, let $\left\{f_{k}\right\}$ be a sequence of smooth functions that converge in the $C^{\infty}$-topology to $f$, and such that for every $k \geqslant 1$ one has $\left\|f_{k}\right\|_{\mathcal{F}, \max } \leqslant C$ for some constant $C$. Then $\|f\|_{\mathcal{F}, \max } \leqslant C$. The fact that $\|\cdot\|_{\mathcal{F}, \max }$ is non-degenerate (i.e., $\|f\|_{\mathcal{F}, \max }=0$ if and only if $f=0$ ) follows from the next lemma.

Lemma 3.3. Let $\mathcal{F} \subset C_{c}^{\infty}(W)$ be a non-empty finite collection of smooth compactly supported functions in $W$. Then, any $\operatorname{Ham}_{c}(W, \omega)$-invariant norm $\|\cdot\|$ on $C_{c}^{\infty}(W)$ which is continuous in the $C^{\infty}$-topology, satisfies $\|\cdot\| \leqslant C\|\cdot\|_{\mathcal{F}, \text { max }}$ for some absolute constant $C$.

Proof of Lemma 3.3. Let $C=\max \{\|g\| ; g \in \mathcal{F}\}$. For any $f=\sum c_{i} \Phi_{i}^{*} f_{i} \in \mathcal{L}_{\mathcal{F}}$, one has:

$$
\|f\| \leq \sum\left|c_{i}\right|\left\|\Phi_{i}^{*} f_{i}\right\| \leq C \sum\left|c_{i}\right| \leq C\|f\|_{\mathcal{F}, \max }
$$

The lemma now follows from combining (3.2), definition (3.1), and the fact that the norm $\|\cdot\|$ is assumed to be continuous in the $C^{\infty}$-topology.

\footnotetext{
${ }^{4}$ When $\|\cdot\|_{\mathcal{F}, \max } \equiv+\infty$, these statements are trivially true.
} 
The following theorem, which is a "local version" of Theorem 1.3, shows that for a suitable choice of a collection $\mathcal{F}$, the subspace $\mathcal{L}_{\mathcal{F}} \subset C_{c}^{\infty}(W)$ is dense in the $C^{\infty}$-topology, and moreover, that the norm $\|\cdot\|_{\mathcal{F}, \max }$ on $C_{c}^{\infty}(W)$ is dominated from above by the $\|\cdot\|_{\infty}$-norm.

Theorem 3.4. There is a finite collection $\mathcal{F} \subset C_{c}^{\infty}(W)$, such that $\|\cdot\|_{\mathcal{F}, \max }$ is a genuine norm on $C_{c}^{\infty}(W)$, and $\|\cdot\|_{\mathcal{F}, \max } \leq C\|\cdot\|_{\infty}$ for some absolute constant $C$.

The remainder of this section is devoted to the proof of Theorem 3.4, which we split into two separate cases:

\subsection{Theorem 3.4 - the two-dimensional case}

We assume that $n=1$, and hence $W=(-L, L) \times(-L, L)$. We set $z=x+i y$, where $\{x, y\}$ are local coordinates on $W$, and denote by $D_{a}=\{|z| \leq a\}$ the disc with radius $a$ centered at the origin, and by $D_{a, A}=\{a \leq|z| \leq A\}$ the annulus with radii $a, A$. The proof of Theorem 3.4 in the two-dimensional case follows from the next two propositions, the proof of which we postpone to Subsections 3.1.1 and 3.1.2.

Proposition 3.5. There are positive constants a, $A, C$ such that $a<A<L$; a smooth radial function $\mathfrak{f}_{1}$ with $\operatorname{supp}\left(\mathfrak{f}_{1}\right)=D_{A}$; and an integer number $N_{0} \in \mathbb{N}$, such that every $f \in C_{c}^{\infty}(W)$ with $\|f\|_{\infty} \leqslant 1$ can be decomposed as

$$
f=\sum_{j=1}^{N_{0}} \epsilon_{j} \Phi_{j}^{*} g_{j}
$$

where $\Phi_{j} \in \operatorname{Ham}_{c}(W, \omega), \epsilon_{j} \in\{-1,1\}$, and $g_{j}$ are smooth radial functions that satisfy:

$$
\operatorname{supp}\left(g_{j}\right)=D_{A}, g_{j} \equiv \mathfrak{f}_{1} \text { on } D_{a}, \text { and }\left\|g_{j}\right\|_{\infty} \leqslant C
$$

Proposition 3.6. Let $0<a<A$ be positive numbers. Then there exists a smooth function $F_{a, A}: \mathbb{R}^{2} \rightarrow \mathbb{R}$ with $\operatorname{supp}\left(F_{a, A}\right) \subset D_{A}$, such that the following holds: for every smooth radial function $f: \mathbb{R}^{2} \rightarrow \mathbb{R}$, that satisfies

$$
\|f\|_{\infty} \leqslant 1, \operatorname{supp}(f) \subset D_{a, A}, \text { and } \int_{\mathbb{R}^{2}} f \omega=0
$$

there exists an area-preserving diffeomorphism $\Phi: \mathbb{R}^{2} \rightarrow \mathbb{R}^{2}$, with $\operatorname{supp}(\Phi) \subset D_{a, A}$, and such that:

$$
\int_{D_{r}} \Phi^{*} F_{a, A} \omega=\int_{D_{r}} f \omega, \text { for any } r>0
$$

We are now in a position to prove Theorem 3.4 in the two-dimensional case. 
Proof of Theorem 3.4 (the 2-dimensional case): Let $f \in C_{c}^{\infty}(W)$ with $\|f\|_{\infty} \leqslant 1$. It follows from Proposition 3.5 above that there are positive constants $a, A, C$, an integer $N_{0}$, and a smooth radial function $\mathfrak{f}_{1}$ with $\operatorname{supp}\left(\mathfrak{f}_{1}\right)=D_{A}$, such that $f$ can be written as

$$
f=\sum_{j=1}^{N_{0}} \epsilon_{j} \Phi_{j}^{*} g_{j}
$$

where $\Phi_{j} \in \operatorname{Ham}_{c}(W, \omega), \epsilon_{j} \in\{-1,1\}$, and $\left\{g_{j}\right\}$ are smooth radial functions that satisfy (3.1.3. Next, let $\mathfrak{f}_{2}$ be a smooth radial function with $\operatorname{supp}\left(\mathfrak{f}_{2}\right)=D_{a, A}$ such that $\int_{W^{2}} \mathfrak{f}_{2} \omega=1$. Moreover, let $\mathfrak{f}_{0}=F_{a, A}$ be the function provided by Proposition 3.6 above. We consider the function

$$
h_{j}:=g_{j}-\mathfrak{f}_{1}-c_{j} \mathfrak{f}_{2}, \text { where } c_{j}=\int_{W}\left(g_{j}-\mathfrak{f}_{1}\right) \omega
$$

Note that there exists a constant $C^{\prime}$ such that $\left\|h_{j}\right\|_{\infty} \leq C^{\prime}$. Indeed:

$$
\left\|h_{j}\right\|_{\infty} \leq C+\left\|\mathfrak{f}_{1}\right\|_{\infty}+\left|c_{j}\right|\left\|\mathfrak{f}_{2}\right\|_{\infty} \leq C+\left\|\mathfrak{f}_{1}\right\|_{\infty}+\left\|\mathfrak{f}_{2}\right\|_{\infty}\left(\pi C A^{2}+\int_{W}\left|\mathfrak{f}_{1}\right| \omega\right)
$$

From Proposition 3.6 it follows that there are area-preserving diffeomorphisms $\widetilde{\Phi}_{j}$ with $\operatorname{supp}\left(\widetilde{\Phi}_{j}\right) \subset D_{a, A}$, such that for any $r>0$ one has

$$
\int_{D_{r}}\left(\widetilde{\Phi}_{j}^{*} \mathfrak{f}_{0}\right) \omega=\frac{1}{C^{\prime}} \int_{D_{r}} h_{j} \omega
$$

To complete the proof of the theorem, we shall need the following technical lemma:

Lemma 3.7. Let $f \in C_{c}^{\infty}(D)$ be a compactly supported function in a disk $D$. Then

$$
\frac{1}{N} \sum_{i=1}^{N} f\left(z e^{\frac{2 \pi i}{N}}\right) \stackrel{N \rightarrow \infty}{\longrightarrow} \frac{1}{2 \pi} \int_{0}^{2 \pi} f\left(z e^{i \theta}\right) d \theta, \quad \text { in the } C^{\infty} \text { topology }
$$

Postponing the proof of Lemma 3.7, we first finish the proof of the theorem.

Consider a compactly supported Hamiltonian isotopy $T_{\theta}^{A}: W \rightarrow W$, where $\theta \in \mathbb{R}$, and such that $T_{\theta}^{A}(z)=e^{i \theta} z$ in $D_{A}$. From Lemma 3.7 and (3.1.5 it follows that:

$$
\frac{C^{\prime}}{N} \sum_{k=1}^{N}\left(T_{\frac{2 \pi k}{N}}^{A}\right)^{*} \widetilde{\Phi}_{j}^{*} \mathfrak{f}_{0} \stackrel{N \rightarrow \infty}{\longrightarrow} h_{j}, \quad \text { in the } C^{\infty} \text { topology }
$$

We set $\mathcal{F}=\left\{\mathfrak{f}_{0}, \mathfrak{f}_{1}, \mathfrak{f}_{2}\right\}$. From (3.1.6) and Remark 3.2 it follows that $\left\|h_{j}\right\|_{\mathcal{F}, \max } \leq C^{\prime}$. Moreover, by definition one has: $\left\|\mathfrak{f}_{1}\right\|_{\mathcal{F}, \max },\left\|\mathfrak{f}_{2}\right\|_{\mathcal{F}, \max } \leq 1$. This implies that

$$
\left\|g_{j}\right\|_{\mathcal{F}, \max } \leq C^{\prime \prime}
$$


where $C^{\prime \prime}$ is an absolute constant given by:

$$
C^{\prime \prime}=C^{\prime}+1+\pi C A^{2}+\int_{W}\left|\mathfrak{f}_{1}\right| \omega
$$

Thus, we conclude that $\|f\|_{\mathcal{F}, \max } \leq N_{0} C^{\prime \prime}$. This completes the proof of the theorem.

Proof of Lemma 3.7. We shall prove the convergence

$$
\frac{1}{N} \sum_{i=1}^{N} f\left(z e^{\frac{2 \pi i}{N}}\right) \stackrel{N \rightarrow \infty}{\longrightarrow} \frac{1}{2 \pi} \int_{0}^{2 \pi} f\left(z e^{i \theta}\right) d \theta
$$

in $C_{c}^{k}(D)$, for any $k \in \mathbb{N}$. Note that the operators $P_{N}(f)=\frac{1}{N} \sum_{i=1}^{N} f\left(z e^{\frac{2 \pi i}{N}}\right)$, defined on the space $C_{c}^{k}(D)$, have a bounded operator norm which is independent on $N$. Therefore, it is enough to check that

$$
P_{N} f \stackrel{N \rightarrow \infty}{\longrightarrow} \frac{1}{2 \pi} \int_{0}^{2 \pi} f\left(z e^{i \theta}\right) d \theta,
$$

in $C_{c}^{k}(D)$ only on some dense subspace. We choose this subspace to be consists of all the finite sums:

$$
s_{m}(z)=\sum_{l=0}^{m} u_{l}(r) \cos (l \theta)+v_{l}(r) \sin (l \theta),
$$

where $u_{l}$ and $v_{l}$ are smooth radial functions supported in the disk $D$. Note that for $N>m$ one has

$$
P_{N} s_{m}(z)=u_{0}(r)=\frac{1}{2 \pi} \int_{0}^{2 \pi} s_{m}\left(z e^{i \theta}\right) d \theta,
$$

and hence the statement of the lemma is satisfied in a trivial way. The proof of the lemma is now complete.

We now return to complete the proof of Proposition 3.5 and Proposition [3.6.

\subsubsection{Proof of Proposition 3.5}

For the sake of clarity, we fragment the proof of the proposition in several steps:

Step I: We choose $a=\frac{L}{4}, A=\frac{L}{2}$. The area of the sector

$$
\left\{z \in W|a<| z \mid<A ; 0<\operatorname{Arg} z<\frac{\pi}{2}\right\}
$$

equals to

$$
\frac{\pi}{4}\left(\frac{L^{2}}{4}-\frac{L^{2}}{16}\right)=\frac{3 \pi}{64} L^{2}>\frac{L^{2}}{8}=\frac{\operatorname{Area}(W)}{32}
$$


Using a smooth partition of unity, one can decompose $f$ as $f=\sum_{k=1}^{33} f_{k}$, where the support of each $f_{k}$ lies in an open sub-rectangle of the square $W$ of area $\frac{\operatorname{Area}(W)}{32}$, and $\left\|f_{k}\right\|_{\infty} \leq 1$. Next, we take compactly supported area-preserving diffeomorphisms $\widetilde{\Phi}_{k}: W \rightarrow W$, such that $f_{k}=\widetilde{\Phi}_{k}^{*} f_{k}^{\prime}$, for $k=1, \ldots, 33$, and $\operatorname{supp}\left(f_{k}^{\prime}\right) \subset\left(0, \frac{L}{4}\right) \times\left(0, \frac{L}{2}\right)$. Denote $L_{1}=\frac{L}{4}$ and $L_{2}=\frac{L}{2}$. From the above we conclude that it is enough to restrict ourselves to the case where $\operatorname{supp}(f) \subset\left(0, L_{1}\right) \times\left(0, L_{2}\right)$. Indeed, if the proposition holds for such functions, then by replacing $N_{0}$ with $33 N_{0}$, it will hold for any compactly supported function $f \in C_{c}^{\infty}(W)$.

Step II: Following Step I, we assume that $\operatorname{supp}(f) \subset\left(0, L_{1}\right) \times\left(0, L_{2}\right)$. Next, we apply the following lemma to the function $f$.

Lemma 3.8. Let $R=\left[0, L_{1}\right] \times\left[0, L_{2}\right] \subset \mathbb{R}^{2}$ be a rectangle, and let $f: \mathbb{R}^{2} \rightarrow \mathbb{R}$ be a smooth function with $\operatorname{supp}(f) \subset \operatorname{int}(R)$, and $\|f\|_{\infty} \leqslant 1$. Then there exists a decomposition $f=\sum_{i=1}^{8} f_{i}$, and compactly supported diffeomorphisms $\Psi_{i}: R \rightarrow R$, $i=1,2, \ldots, 8$, such that the functions $g_{i}:=\Psi_{i}^{*} f_{i}$ satisfy $\left|\frac{\partial}{\partial x} g_{i}\right| \leqslant \frac{12}{L_{1}}$.

The proof of Lemma 3.8 will be given in Subsection 3.1.3.

Remark 3.9. Analogously to Step I, Lemma 3.8 reduces the proposition to the case where $\operatorname{supp}(f) \subset\left(0, L_{1}\right) \times\left(0, L_{2}\right)$, and moreover that there is a diffeomorphism $\Psi: W \rightarrow W$ with $\operatorname{supp}(\Psi) \subset\left(0, L_{1}\right) \times\left(0, L_{2}\right)$, such that $g=\Psi^{*} f$ satisfies $\left|\frac{\partial}{\partial x} g\right| \leqslant \frac{12}{L_{1}}$. Indeed, the general case would follow by replacing $N_{0}$ with $8 \cdot 33 \cdot N_{0}=264 N_{0}$. Thus, we assume in what follows the existence of $f, g$ and $\Psi$ as above.

Step III: Denote by $\mathcal{R}$ the rectangle $\left[0, L_{1}\right] \times\left[0, L_{2}\right]$. From the fact that

$$
\operatorname{Area}(\mathcal{R})<\operatorname{Area}\left(\left\{z \in W|a<| z \mid<A ; 0<\operatorname{Arg} z<\frac{\pi}{2}\right\}\right)
$$

one can easily find an area preserving diffeomorphism $\Phi: W \rightarrow W$ with

$$
\Phi(\mathcal{R})=\left\{z \in W|a<| z \mid<A_{1} ; 0<\operatorname{Arg} z<\frac{\pi}{2}\right\}
$$

for an appropriate $a<A_{1}<A$; and such that on $\mathcal{R}$, the diffeomorphism $\Phi$ takes the form $\Phi(x+i y)=r_{1}(x) e^{\theta_{1}(y)}$, where $r_{1}(x)$ is a monotone increasing function. Let $C_{1}=\min _{x \in\left[0, L_{1}\right]} r_{1}^{\prime}(x)>0$, and define $h=\left(\Phi^{-1}\right)^{*} g$. Note that one can bound the radial derivative of $h$ by:

$$
\max \left|\frac{\partial}{\partial r} h\right| \leq \frac{1}{C_{1}} \max \left|\frac{\partial}{\partial x} g\right| \leq \frac{12}{L_{1} C_{1}}
$$

Next, we set $C_{2}=\frac{12}{L_{1} C_{1}}$, and fix a smooth radial function $\mathfrak{f}_{1}$ such that

$$
\operatorname{supp}\left(\mathfrak{f}_{1}\right) \subset D_{A}, \quad \frac{\partial}{\partial r} \mathfrak{f}_{1}(z)<-C_{2} \text { for } z \in D_{a, A_{1}}, \quad \frac{\partial}{\partial r} \mathfrak{f}_{1}(z)<0 \text { for } z \in \operatorname{int}\left(D_{A}\right) \backslash\{0\},
$$


and such that the point $z=0$ is a non-degenerate maximum for the function $\mathfrak{f}_{1}$. We denote $H=h+\mathfrak{f}_{1}(z)$, and observe that $H$ satisfies:

$$
\operatorname{supp}(H) \subset D_{A}, \quad \frac{\partial}{\partial r} H<0 \text { in } \operatorname{int}\left(D_{A}\right) \backslash\{0\}, \quad H(z) \equiv \mathfrak{f}_{1}(z) \text { in } D_{a} \cup D_{A_{1}, A},
$$

and that the point $z=0$ is a unique non-degenerate critical point of $H$, which is a maximum point. Consider the gradient flow of $H$. By a standard Morse theory argument one can find a diffeomorphism $\Upsilon: W \rightarrow W$, with $\operatorname{supp}(\Upsilon) \subset D_{a, A}$, and such that $K:=\Upsilon^{*} H$ is a radial function. Finally, we have

$$
\begin{aligned}
f & =\left(\Psi^{-1}\right)^{*} g=\left(\Psi^{-1}\right)^{*} \Phi^{*} h=\left(\Psi^{-1}\right)^{*} \Phi^{*} H-\left(\Psi^{-1}\right)^{*} \Phi^{*} \mathfrak{f}_{1} \\
& =\left(\Psi^{-1}\right)^{*} \Phi^{*}\left(\Upsilon^{-1}\right)^{*} K-\left(\Psi^{-1}\right)^{*} \Phi^{*} \mathfrak{f}_{1} .
\end{aligned}
$$

Note, that for $z \in W \backslash D_{a, A}$, one has

$$
\Psi \Phi^{-1} \Upsilon(z)=\Psi \Phi^{-1}(z)=\Phi^{-1}(z)
$$

Indeed, this follows from the fact that $\operatorname{supp}(\Psi) \subset \mathcal{R} \subset \Phi^{-1}\left(D_{a, A}\right)$, and that $\Upsilon$ is the identity on the complement $W \backslash D_{a, A}$. Thus, we conclude that

$$
\left(\Psi \Phi^{-1} \Upsilon\right)^{*} \omega=\left(\Psi \Phi^{-1}\right)^{*} \omega=\omega, \text { on the complement } W \backslash D_{a, A}
$$

Next, let $S_{r}=\{z \in W|| z \mid=r\}$. We shall need the following lemma:

Lemma 3.10. Let $\omega^{\prime}$ be a symplectic form on $W$ which coincides with the standard symplectic form $\omega$ on the complement $W \backslash D_{a, A}$, and such that $\int_{W} \omega^{\prime}=\int_{W} \omega$. Then, there exists a diffeomorphism $\Lambda: W \rightarrow W$ supported in $D_{a, A}$, such that for every $a<r<A$, one has $\Lambda\left(S_{r}\right)=S_{R}$, for some $a<R<A$, and such that $\Lambda^{*} \omega=\omega^{\prime}$.

Proof of Lemma 3.10. Consider the function $S:[0, L) \rightarrow[0, \infty)$, defined by $S(r)=\int_{D_{r}} \omega^{\prime}$. Note that $S$ is a smooth function, and that $S(r)=\pi r^{2}$ for every $r \in[0, a] \cup[A, L)$. Define a diffeomorphism $\Delta_{1}: W \rightarrow W$, supported in $D_{a, A}$, by

$$
\Delta_{1}(r, \theta)=\left(\sqrt{\frac{S(r)}{\pi}}, \theta\right), \text { for } r \in[0, L),
$$

and extend it by the identity diffeomorphism to the whole $W$. Denote $\omega^{\prime \prime}=\left(\Delta_{1}^{-1}\right)^{*} \omega^{\prime}$, and note that $\int_{D_{r}} \omega^{\prime \prime}=\pi r^{2}$ for $r \leqslant A$, and $\omega^{\prime \prime}=\omega^{\prime}=\omega$ on $W \backslash D_{a, A}$. Next, we explicitly construct a diffeomorphism $\Delta_{2}: W \rightarrow W$ supported in $D_{a, A}$, such that $\omega^{\prime \prime}=\Delta_{2}^{*} \omega$, and for $0<r<L$, it takes the form $\Delta_{2}(r, \theta)=(r, F(r, \theta))$, for some smooth map $F:(0, L) \times S^{1} \rightarrow S^{1}$. To this end, note that $\omega^{\prime \prime}=G \omega$ for some positive function $G: W \rightarrow(0, \infty)$, such that $G=1$ on $W \backslash D_{a, A}$. Moreover,

$$
\pi r^{2}=\int_{D_{r}} \omega^{\prime \prime}=\int_{D_{r}} G \omega, \text { for all } 0<r<L
$$


After differentiating this equality we obtain

$$
\int_{0}^{2 \pi} G(r, \theta) d \theta=2 \pi, \text { for every } 0<r<L
$$

On the other hand, we require $\Delta_{2}$ to satisfy:

$$
\Delta_{2}^{*} \omega=r F_{\theta}(r, \theta) d r \wedge d \theta=F_{\theta}(r, \theta) \omega, \text { for every } r \in(0, L)
$$

Thus, the condition $\omega^{\prime \prime}=\Delta_{2}^{*} \omega$ is equivalent to $F_{\theta}(r, \theta)=G(r, \theta)$, for $r \in(0, L)$. We define

$$
F(r, \theta)=\int_{0}^{\theta} G(r, s) d s, \text { for } r \in(0, L), \theta \in[0,2 \pi)
$$

In light of (3.1.7), we obtain a smooth map $F:(0, L) \times S^{1} \rightarrow S^{1}$. Moreover, since $G=1$ on $W \backslash D_{a, A}$, one has $F(r, \theta)=\theta$ for $r \in(0, a] \cap[A, L)$. Therefore, defining $\Delta_{2}(r, \theta)=(r, F(r, \theta))$ for $0<r<L$, where $F$ is given in (3.1.8), we obtain a diffeomorphism of $D_{L}$ supported in $D_{a, A}$. We extend $\Delta_{2}$ to the whole $W$ by the identity diffeomorphism. Note that $\omega^{\prime \prime}=\Delta_{2}^{*} \omega$, and hence $\omega^{\prime}=\Delta_{1}^{*} \omega^{\prime \prime}=\Delta_{1}^{*} \Delta_{2}^{*} \omega$. Denoting $\Lambda=\Delta_{2} \Delta_{1}$, we conclude the statement of the lemma.

We return now to the proof of the Proposition. By applying Lemma 3.10 to the forms $\omega^{\prime}=\left(\Psi \Phi^{-1} \Upsilon\right)^{*} \omega$ and $\omega^{\prime \prime}=\left(\Psi \Phi^{-1}\right)^{*} \omega$, we obtain two diffeomorphisms $\Lambda^{\prime}, \Lambda^{\prime \prime}$ such that $\Lambda^{\prime *} \omega=\left(\Psi \Phi^{-1} \Upsilon\right)^{*} \omega$, and $\Lambda^{\prime \prime *} \omega=\left(\Psi \Phi^{-1}\right)^{*} \omega$. Denote $\Phi^{\prime}:=\Lambda^{\prime} \Upsilon^{-1} \Phi \Psi^{-1}$, $\Phi^{\prime \prime}:=\Lambda^{\prime \prime} \Phi \Psi^{-1}$. Note that $\Phi^{\prime}, \Phi^{\prime \prime} \in \operatorname{Ham}_{c}(W, \omega)$, and that

$$
\begin{aligned}
f & =\left(\Psi^{-1}\right)^{*} \Phi^{*}\left(\Upsilon^{-1}\right)^{*} K-\left(\Psi^{-1}\right)^{*} \Phi^{*} \mathfrak{f}_{1}=\left(\Psi^{-1}\right)^{*} \Phi^{*}\left(\Upsilon^{-1}\right)^{*}\left(\Lambda^{\prime}\right)^{*} K-\left(\Psi^{-1}\right)^{*} \Phi^{*}\left(\Lambda^{\prime \prime}\right)^{*} \mathfrak{f}_{1} \\
& =\left(\Phi^{\prime}\right)^{*} K-\left(\Phi^{\prime \prime}\right)^{*} \mathfrak{f}_{1}
\end{aligned}
$$

The decomposition $f=\left(\Phi^{\prime}\right)^{*} K-\left(\Phi^{\prime \prime}\right)^{*} \mathfrak{f}_{1}$ shows that the proposition holds for $f$ as in Remark 3.9, with only two summands in the decomposition, and with $C=\left\|\mathfrak{f}_{1}\right\|_{\infty}$. Therefore, we obtain the conclusion of Proposition 3.5 with $N_{0}=264 \cdot 2=528$.

\subsubsection{Proof of Proposition 3.6}

We start with a construction of a function $F$, such that for any smooth radial function $f: \mathbb{R}^{2} \rightarrow \mathbb{R}$, satisfying the conditions 3.1 .4 one can find a diffeomorphism (not necessarily area-preserving) $\Psi: \mathbb{R}^{2} \rightarrow \mathbb{R}^{2}$ supported in $D_{A}$ such that for any $r>0$ :

$$
\int_{D_{r}} \Psi^{*} \omega=\int_{D_{r}} \omega=\pi r^{2}
$$

and,

$$
\int_{D_{r}} \Psi^{*}(F \omega)=\int_{D_{r}} f \omega
$$


We shall take the function $F$ to be of the form $F(r, \theta)=\phi(r) \psi(\theta)$, where $\phi, \psi$ are smooth functions. We assume that $\phi(r)=0$, for small enough $r$, and that $\phi(r)=1$ for $r \geqslant a$. The function $\psi$ is assumed to satisfy $\int_{0}^{2 \pi} \psi(\theta) d \theta=0$, and would be determined in the sequel. Moreover,

$$
\left\{\begin{array}{l}
R(r, \theta)=\sqrt{u(r) \mu(\theta)+v(r) \nu(\theta)}, \\
u(r)=v(r)=r^{2} \text { for } r \leqslant a \text { or } r \geqslant A, \\
u^{\prime}(r), v^{\prime}(r)>0 \text { for } r>0, \\
\mu(\theta), \nu(\theta) \geqslant 0, \\
\mu(\theta)+\nu(\theta)=1
\end{array}\right.
$$

Here, $\mu, \nu, u$ and $v$, are smooth functions that would be determined explicitly in the sequel. Note that conditions (3.1.11) ensure that $\Psi$ is a diffeomorphism of $\mathbb{R}^{2}$ supported in $D_{a, A}$. Next, we compute

$$
\Psi^{*} \omega=R(r, \theta) R_{r}^{\prime}(r, \theta) d r \wedge d \theta=\frac{1}{2}\left(u^{\prime}(r) \mu(\theta)+v^{\prime}(r) \nu(\theta)\right) d r \wedge d \theta,
$$

and

$$
\begin{aligned}
\Psi^{*}(F \omega) & =F(R(r, \theta), \theta) R(r, \theta) R_{r}^{\prime}(r, \theta) d r \wedge d \theta \\
& =\frac{1}{2} \phi(R(r, \theta)) \psi(\theta)\left(u^{\prime}(r) \mu(\theta)+v^{\prime}(r) \nu(\theta)\right) d r \wedge d \theta
\end{aligned}
$$

After differentiating by $r$ and some simplification, conditions (3.1.9), (3.1.10) become

$$
u^{\prime}(r) \int_{0}^{2 \pi} \mu(\theta) d \theta+v^{\prime}(r) \int_{0}^{2 \pi} \nu(\theta) d \theta=4 \pi r
$$

and,

$$
u^{\prime}(r) \int_{0}^{2 \pi} \phi(R(r, \theta)) \psi(\theta) \mu(\theta) d \theta+v^{\prime}(r) \int_{0}^{2 \pi} \phi(R(r, \theta)) \psi(\theta) \nu(\theta) d \theta=4 \pi r f(r)
$$

Note that when $r \geqslant a$, one has $R(r, \theta) \geqslant a$, and condition (3.1.13 turns to:

$$
u^{\prime}(r) \int_{0}^{2 \pi} \psi(\theta) \mu(\theta) d \theta+v^{\prime}(r) \int_{0}^{2 \pi} \psi(\theta) \nu(\theta) d \theta=4 \pi r f(r)
$$

Next, we choose the functions $\psi, \mu, \nu$ to be any smooth functions satisfying:

$$
\left\{\begin{array}{l}
\int_{0}^{2 \pi} \psi(\theta) \mu(\theta) d \theta=2 \pi \\
\int_{0}^{2 \pi} \psi(\theta) \nu(\theta) d \theta=-2 \pi \\
\int_{0}^{2 \pi} \mu(\theta) d \theta=\int_{0}^{2 \pi} \nu(\theta) d \theta=\pi \\
\mu(\theta), \nu(\theta) \geqslant 0 \\
\mu(\theta)+\nu(\theta)=1
\end{array}\right.
$$


Note that this choice of $\psi, \mu, \nu$ do not depend on the function $f$. Moreover, with the above choice, for $r \geqslant a$, equations (3.1.12) and (3.1.14 become

$$
\left\{\begin{array}{l}
u^{\prime}(r)+v^{\prime}(r)=4 r \\
u^{\prime}(r)-v^{\prime}(r)=2 r f(r)
\end{array}\right.
$$

Next, we consider equations (3.1.16 for every $r \geqslant 0$, with initial conditions $u(0)=$ $v(0)=0$. There is no difficulty in checking that the solutions of this system are

$$
\left\{\begin{array}{l}
u(r)=\int_{0}^{r} s(2+f(s)) d s \\
v(r)=\int_{0}^{r} s(2-f(s)) d s
\end{array}\right.
$$

One can easily check, that as required, the function $u$ and $v$ satisfy

$$
\left\{\begin{array}{l}
u^{\prime}(r), v^{\prime}(r)>0, \text { for } r>0, \\
u(r)=v(r)=r^{2}, \text { for } r \leqslant a \text { and } r \geqslant A
\end{array}\right.
$$

Moreover, by definition, they satisfy equations (3.1.12) and (3.1.13) when $r \geqslant a$. Let us now show that these equations hold for $r<a$ as well. First, note that equation (3.1.12 clearly holds when $r<a$. Second, by defintion, for $r<a$ one has $u(r)=v(r)=r^{2}$, and $R(r, \theta)=r$. Hence, we compute

$$
\begin{gathered}
u^{\prime}(r) \int_{0}^{2 \pi} \phi(R(r, \theta)) \psi(\theta) \mu(\theta) d \theta+v^{\prime}(r) \int_{0}^{2 \pi} \phi(R(r, \theta)) \psi(\theta) \nu(\theta) d \theta \\
=u^{\prime}(r) \phi(r) \int_{0}^{2 \pi} \psi(\theta) \mu(\theta) d \theta+v^{\prime}(r) \phi(r) \int_{0}^{2 \pi} \psi(\theta) \nu(\theta) d \theta \\
=2 r \phi(r)\left(\int_{0}^{2 \pi} \psi(\theta) \mu(\theta) d \theta+\int_{0}^{2 \pi} \psi(\theta) \nu(\theta) d \theta\right) \\
=2 r \phi(r)(2 \pi-2 \pi)=0
\end{gathered}
$$

Combining this with the fact that $\operatorname{supp}(f) \subset D_{a, A}$, we obtain that the functions $u$ and $v$, satisfy 3.1 .12 and 3.1 .13 for all $r \geqslant 0$. We conclude that the resulting diffeomorphism $\Psi$ satisfies conditions (3.1.9) and (3.1.10). Furthermore, since the diffeomorphism $\Psi$ satisfies (3.1.9), and $\operatorname{supp}(\Psi) \subset D_{a, A}$, by using a similar arguments as in the proof of Lemma (3.1.5) from [16], we conclude that there exists an areapreserving diffeomorphism $\Phi: \mathbb{R}^{2} \rightarrow \mathbb{R}^{2}$, with $\operatorname{supp}(\Phi) \in D_{a, A}$, such that $\Phi(D(r))=$ $\Psi(D(r))$ for any $r>0$. Thus, we obtain

$$
\int_{D_{r}}\left(\Phi^{*} F\right) \omega=\int_{D_{r}} \Phi^{*}(F \omega)=\int_{\Phi\left(D_{r}\right)} F \omega=\int_{\Psi\left(D_{r}\right)} F \omega=\int_{D_{r}} \Psi^{*}(F \omega)=\int_{D_{r}} f \omega
$$

and the proof of the Proposition in now complete. 


\subsubsection{Technical Lemmata}

In this subsection we prove Lemma 3.8 which was used in the proof of Proposition 3.5. We start with the following preparation:

Lemma 3.11. There is a smooth function $\phi: \mathbb{R} \rightarrow \mathbb{R}$ with the following properties:

1. $\operatorname{supp}(\phi)=[0,3]$,

2. $\phi(t)>0$, for $t \in(0,3)$,

3. $\phi^{\prime}(t)>0$, for $t \in(0,3 / 2)$, and $\phi^{\prime}(t)<0$ for $t \in(3 / 2,3)$,

4. $\sup _{t \in(0,3)}\left(\frac{\phi^{\prime}(t)}{\phi(t)}\right)^{\prime}=\sup _{t \in(0,3)} \frac{\phi^{\prime \prime}(t) \phi(t)-\phi^{\prime}(t)^{2}}{\phi(t)^{2}}<0$,

5. $\sum_{n \in \mathbb{Z}} \phi(t+n) \equiv 1$

Proof of Lemma 3.11. Consider first the smooth function $f: \mathbb{R} \rightarrow \mathbb{R}$, defined by

$$
f(x)= \begin{cases}e^{-\frac{2}{x}}, & \text { for } x>0 \\ 0, & \text { for } x \leqslant 0\end{cases}
$$

Note that for $x>0$, one has

$$
f^{\prime \prime}(x)=\frac{4}{x^{4}} e^{-\frac{2}{x}}(1-x),
$$

and hence $f^{\prime \prime}(x)>0$ for $x \in(0,1)$, and $f^{\prime \prime}(0)=f^{\prime \prime}(1)=0$. Note moreover that

$$
f^{\prime \prime}(x) f(x)-f^{\prime}(x)^{2}=\left(\frac{f^{\prime}(x)}{f(x)}\right)^{\prime} f(x)^{2}=-\frac{4}{x^{3}} e^{-\frac{4}{x}}<0, \text { for } x \in(0,+\infty)
$$

We approximate, in the $C^{0}$-norm, the function $\left.f^{\prime \prime}\right|_{[0,1]}$ arbitrarily close by a smooth positive function $h:[0,1] \rightarrow[0, \infty)$, such that $h(x)=f^{\prime \prime}(x)$ for $x \in\left[0, \frac{1}{2}\right]$, and such that $h(x)=0$ near $x=1$. Next, consider the smooth function $F:[0,1] \rightarrow \mathbb{R}$, that is uniquely determined by the requirements $F^{\prime \prime}(x)=h(x)$, and $F(0)=F^{\prime}(0)=0$. Note that the function $F$ is arbitrary close, in the $C^{2}$-topology, to $\left.f\right|_{[0,1]}$, and $F(x)=f(x)$ for $x \in\left[0, \frac{1}{2}\right]$. Moreover, the requirement that $h$ is $C^{0}$-sufficiently close to $\left.f^{\prime \prime}\right|_{[0,1]}$ ensures that $F^{\prime \prime}(x) F(x)-F^{\prime}(x)^{2}<0$, for every $x \in(0,1)$. We further observe that by definition, $F^{\prime \prime}(x)+F^{\prime \prime}(1-x)>0$ for all $x \in(0,1)$, and that $F(x)$ is a linear function near $x=1$. Finally, we define $\phi: \mathbb{R} \rightarrow \mathbb{R}$ as follows:

$$
\phi(x)= \begin{cases}\frac{F(x)}{2 F(1)} & \text { for } x \in[0,1], \\ \frac{2 F(1)-F(x-1)-F(2-x)}{2 F(1)} & \text { for } x \in(1,2], \\ \frac{F(3-x)}{2 F(1)} & \text { for } x \in(2,3], \\ 0 & \text { for } x \notin[0,3]\end{cases}
$$


It follows immediately from the definition that $\phi$ is a non-negative smooth function, with $\operatorname{supp}(\phi)=[0,3]$. Note moreover that $\phi(x)=\phi(3-x)$, and that for $x \in(1,2)$ :

$$
\left(\phi_{\left.\right|_{(1,2)}}\right)^{\prime \prime}(x)=\frac{-F^{\prime \prime}(x-1)-F^{\prime \prime}(2-x)}{2 F(1)}<0
$$

Combining this with the fact that $\phi^{\prime}(3 / 2)=0$, we obtain that $\phi^{\prime}(x)>0$ for $x \in$ $(1,3 / 2)$, and $\phi^{\prime}(x)<0$ for $x \in(3 / 2,3)$. Furthermore, from the definition of the function $F$, it follows that $\phi^{\prime}(x)>0$ for $x \in(0,1]$ and $\phi^{\prime}(x)<0$ for $x \in[2,3)$. Thus, we conclude that $\phi$ satisfies the first three requirements of the lemma. We next turn to show that $\phi$ satisfies the forth one. Note that $\phi^{\prime \prime}(x) \phi(x)-\phi^{\prime}(x)^{2}<0$ for $x \in(0,3)$. This follows from the analogous property of $F$ for $x \in(0,1) \cup(2,3)$; from (3.1.19) for $x \in(1,2)$; and from the fact that $\phi^{\prime \prime}\left(x_{0}\right) \phi\left(x_{0}\right)-\phi^{\prime}\left(x_{0}\right)^{2}=-\phi^{\prime}\left(x_{0}\right)^{2}<0$ for $x_{0}=1,2$. Moreover, from the definition of the function $\phi$ it follows that $\phi(x) \simeq e^{-\frac{2}{x}}$ for $x$ close to 0 , and $\phi(x) \simeq e^{-\frac{2}{3-x}}$ for $x$ close to 3 , where $\simeq$ means arbitrary close in the $C^{2}$-topology. Therefore, we obtain:

$$
\lim _{x \rightarrow 0^{+}} \frac{\phi^{\prime \prime}(x) \phi(x)-\phi^{\prime}(x)^{2}}{\phi(x)^{2}}=\lim _{x \rightarrow 3^{-}} \frac{\phi^{\prime \prime}(x) \phi(x)-\phi^{\prime}(x)^{2}}{\phi(x)^{2}}=-\infty .
$$

From the above we conclude that:

$$
\sup _{x \in(0,3)} \frac{\phi^{\prime \prime}(x) \phi(x)-\phi^{\prime}(x)^{2}}{\phi(x)^{2}}<0,
$$

as required. Finally, there is no difficulty in checking that $\sum_{n \in \mathbb{Z}} \phi(x+n)=1$. The details of this last step are left to the reader.

Lemma 3.12. Let $R=\left[\alpha_{1}, \beta_{1}\right] \times\left[\alpha_{2}, \beta_{2}\right] \subset \mathbb{R}^{2}$ be a rectangle, and consider two smooth non-negative functions $u:\left[\alpha_{1}, \beta_{1}\right] \rightarrow \mathbb{R}$, and $v:\left[\alpha_{2}, \beta_{2}\right] \rightarrow \mathbb{R}$, positive on $\left(\alpha_{1}, \beta_{1}\right)$ and $\left(\alpha_{2}, \beta_{2}\right)$ respectively, such that $u(x)=e^{\frac{-1}{x-\alpha_{1}}}$ near $\alpha_{1} ; u(x)=e^{\frac{-1}{\beta_{1}-x}}$ near $\beta_{1}, v(y)=e^{\frac{-1}{y-\alpha_{2}}}$ near $\alpha_{2}$; and $v(y)=e^{\frac{-1}{\beta_{2}-y}}$ near $\beta_{2}$. Moreover, let $\phi(x)$ be the function described in Lemma 3.11 above, and let $F: \mathbb{R}^{2} \rightarrow \mathbb{R}$ be any smooth function that satisfies:

1. $\operatorname{supp}(F)=R$

2. $F(x, y)>0$ for $(x, y) \in \operatorname{int}(R)$

3. $F(x, y)=u(x) v(y)$ near the boundary of $R$

Then there exists an $\epsilon_{0}>0$, such that for any $0<\epsilon<\epsilon_{0}$, and $a \in \mathbb{R}$, the following holds: denote by $G(x, y)=F(x, y) \phi\left(\frac{x-a}{\epsilon}\right)$, and assume that $G \neq 0$ (this holds when $\left.\left(\alpha_{1}, \beta_{1}\right) \cap(a, a+3 \epsilon) \neq \emptyset\right)$. Moreover, set $U=\operatorname{supp}(G)=\left[a_{1}, a_{2}\right] \times\left[\alpha_{2}, \beta_{2}\right]$. Then, 
there exists a smooth function $c:\left[\alpha_{2}, \beta_{2}\right] \rightarrow\left(a_{1}, a_{2}\right)$, which is constant near $\alpha_{2}, \beta_{2}$, such that for any $y \in\left(\alpha_{2}, \beta_{2}\right)$ one has:

$$
\left\{\begin{array}{l}
\frac{\partial}{\partial x} G(x, y)>0, \text { for } a_{1}<x<c(y) \\
\frac{\partial}{\partial x} G(x, y)<0, \text { for } c(y)<x<a_{2}
\end{array}\right.
$$

Proof of Lemma 3.12. From the above assumptions it follows that there exists $\alpha_{1}<\gamma_{1}<\delta_{1}<\beta_{1}$, such that $u(x)=e^{\frac{-1}{x-\alpha_{1}}}$ for $\alpha_{1}<x<\gamma_{1}, u(x)=e^{\frac{-1}{\beta_{1}-x}}$ for $\delta_{1}<x<\beta_{1}$, and $F(x, y)=u(x) v(y)$ when $x \in\left(\alpha_{1}, \gamma_{1}\right] \cup\left[\delta_{1}, \beta_{1}\right)$. Pick some $\gamma_{1}^{\prime}, \delta_{1}^{\prime}$, such that $\alpha_{1}<\gamma_{1}^{\prime}<\gamma_{1}<\delta_{1}<\delta_{1}^{\prime}<\beta_{1}$, and denote $\epsilon_{1}=\min \left\{\frac{\gamma_{1}-\gamma_{1}^{\prime}}{3}, \frac{\delta_{1}^{\prime}-\delta_{1}}{3}\right\}$. Next, take any $0<\epsilon<\epsilon_{1}$, and any $a \in \mathbb{R}$, and consider the function $G(x, y)=F(x, y) \phi\left(\frac{x-a}{\epsilon}\right)$.

Case I: Assume $a \in\left[\gamma_{1}^{\prime}, \delta_{1}\right]$. Then, one has $\gamma_{1}^{\prime} \leqslant a<a+3 \epsilon \leqslant \delta_{1}^{\prime}$, and therefore $\operatorname{supp}(G)=[a, a+3 \epsilon] \times\left[\alpha_{2}, \beta_{2}\right]$. Fix some $y_{0} \in\left(\alpha_{2}, \beta_{2}\right)$. Our goal is to show that for sufficiently small $\epsilon$ (which is independent of $y_{0}$ ), there exists a value $c\left(y_{0}\right) \in(a, a+3 \epsilon)$, such that $\frac{\partial}{\partial x} G\left(x, y_{0}\right)>0$, for $a<x<c\left(y_{0}\right)$, and $\frac{\partial}{\partial x} G\left(x, y_{0}\right)<0$, for $c\left(y_{0}\right)<x<a+3 \epsilon$. For this end, we compute:

$$
\frac{\frac{\partial}{\partial x} G\left(x, y_{0}\right)}{G\left(x, y_{0}\right)}=\frac{\frac{\partial}{\partial x} F\left(x, y_{0}\right)}{F\left(x, y_{0}\right)}+\frac{1}{\epsilon} \frac{\phi^{\prime}\left(\frac{x-a}{\epsilon}\right)}{\phi\left(\frac{x-a}{\epsilon}\right)} .
$$

Note that, the function $x \mapsto G\left(x, y_{0}\right)$ is a positive function, supported in $[a, a+3 \epsilon]$. Thus, $\frac{\frac{\partial}{\partial x} G\left(x, y_{0}\right)}{G\left(x, y_{0}\right)}=0$ at least at one point $x \in(a, a+3 \epsilon)$ (e.g., at the maximum point of $\left.x \mapsto G\left(x, y_{0}\right)\right)$. Let us show next that:

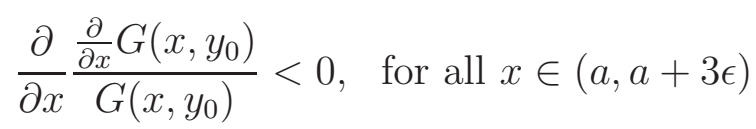

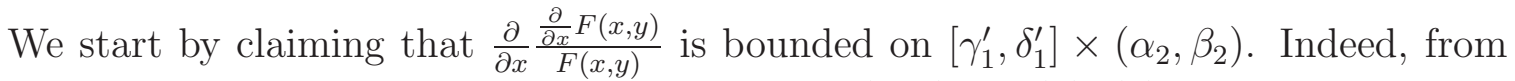
the assumptions of the lemma it follows that $F(x, y)=u(x) v(y)$ near the boundary of $R$, and therefore there exist $\alpha_{2}<\gamma_{2}<\delta_{2}<\beta_{2}$, such that $F(x, y)=u(x) v(y)$ for $y \in\left[\alpha_{2}, \gamma_{2}\right] \cup\left[\delta_{2}, \beta_{2}\right]$. Thus, for a point $(x, y)$ near the boundary of $R$, one has

$$
\frac{\partial}{\partial x} \frac{\frac{\partial}{\partial x} F(x, y)}{F(x, y)}=\frac{\partial}{\partial x} \frac{u^{\prime}(x)}{u(x)}=\frac{u^{\prime \prime}(x) u(x)-u^{\prime}(x)^{2}}{u(x)^{2}}
$$

Restricting ourselves to the case where $x \in\left[\gamma_{1}^{\prime}, \delta_{1}^{\prime}\right]$ and $y \in\left[\alpha_{2}, \gamma_{2}\right] \cup\left[\delta_{2}, \beta_{2}\right]$, and by noticing that $\left.u\right|_{\left(\alpha_{1}, \beta_{1}\right)}$ is strictly positive smooth function, we obtain that the function $\frac{\partial}{\partial x} \frac{\frac{\partial}{\partial x} F(x, y)}{F(x, y)}$ is bounded on $\left[\gamma_{1}^{\prime}, \delta_{1}^{\prime}\right] \times\left(\left(\alpha_{2}, \gamma_{2}\right] \cup\left[\delta_{2}, \beta_{2}\right)\right)$. On the other hand, because

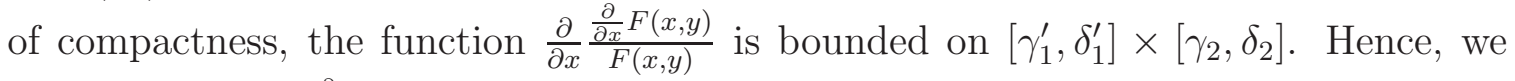
conclude that $\frac{\partial}{\partial x} \frac{\frac{\partial}{\partial x} F(x, y)}{F(x, y)}$ is bounded on $\left[\gamma_{1}^{\prime}, \delta_{1}^{\prime}\right] \times\left(\alpha_{2}, \beta_{2}\right)$. Next, note that

$$
\frac{\partial}{\partial x} \frac{1}{\epsilon} \frac{\phi^{\prime}\left(\frac{x-a}{\epsilon}\right)}{\phi\left(\frac{x-a}{\epsilon}\right)}=\frac{1}{\epsilon^{2}} \frac{\phi^{\prime \prime}\left(\frac{x-a}{\epsilon}\right) \phi\left(\frac{x-a}{\epsilon}\right)-\phi^{\prime}\left(\frac{x-a}{\epsilon}\right)^{2}}{\phi\left(\frac{x-a}{\epsilon}\right)^{2}}
$$


From Lemma 3.11 it follows that

$$
\sup _{t \in(0,3)} \frac{\phi^{\prime \prime}(t) \phi(t)-\phi^{\prime}(t)^{2}}{\phi(t)^{2}}<0
$$

and hence (3.1.22 can be chosen to be arbitrarily negative. As a conclusion, we obtain that for sufficiently small $\epsilon$, say $0<\epsilon<\epsilon_{2}$, one has

$$
\frac{\partial}{\partial x} \frac{\frac{\partial}{\partial x} G(x, y)}{G(x, y)}<0, \text { for every }(x, y) \in \operatorname{supp}(G)=[a, a+3 \epsilon] \times\left(\alpha_{2}, \beta_{2}\right)
$$

Moreover, for any $y \in\left(\alpha_{2}, \beta_{2}\right)$, there exists therefore a unique $x:=c(y) \in(a, a+3 \epsilon)$, such that $\frac{\frac{\partial}{\partial x} G(x, y)}{G(x, y)}=0$. It follows from (3.1.24) and the implicit function theorem, that the function $y \mapsto c(y)$ is smooth for $y \in\left(\alpha_{2}, \beta_{2}\right)$. Moreover, since $\frac{\frac{\partial}{\partial x} G(x, y)}{G(x, y)}$ is independent of $y$, when $y$ is close to $\alpha_{2}$ or to $\beta_{2}$, it follows that $y \mapsto c(y)$ is constant near the endpoints $\alpha_{2}, \beta_{2}$. This completes the proof of the Lemma in Case I.

Case II: Assume that $a<\gamma_{1}^{\prime}$ or $a>\delta_{1}$. Here we have $[a, a+3 \epsilon] \subset\left(-\infty, \gamma_{1}\right) \cup\left(\delta_{1},+\infty\right)$. Therefore, the function

$$
\frac{\frac{\partial}{\partial x} F(x, y)}{F(x, y)}=\frac{u^{\prime}(x)}{u(x)}
$$

is independent of $y$, as well as

$$
\frac{\frac{\partial}{\partial x} G(x, y)}{G(x, y)}=\frac{u^{\prime}(x)}{u(x)}+\frac{1}{\epsilon} \frac{\phi^{\prime}\left(\frac{x-a}{\epsilon}\right)}{\phi\left(\frac{x-a}{\epsilon}\right)},
$$

for $(x, y) \in \operatorname{supp}(G)$. Also, for $(x, y) \in \operatorname{supp}(G)$ one has

$$
\frac{\partial}{\partial x} \frac{\frac{\partial}{\partial x} F(x, y)}{F(x, y)}=\frac{\partial}{\partial x} \frac{u^{\prime}(x)}{u(x)}=\frac{u^{\prime \prime}(x) u(x)-u^{\prime}(x)^{2}}{u(x)^{2}}
$$

Thus, since $u(x)=e^{\frac{-1}{x-\alpha_{1}}}$ for $x \in\left(\alpha_{1}, \gamma_{1}\right)$, and $u(x)=e^{\frac{-1}{\beta_{1}-x}}$ for $x \in\left(\delta_{1}, \beta_{1}\right)$, we obtain

$$
\frac{\partial}{\partial x} \frac{\frac{\partial}{\partial x} F(x, y)}{F(x, y)}<0, \text { for }(x, y) \in \operatorname{supp}(G)
$$

As in Case I, by combining (3.1.22) and (3.1.23), one has

$$
\frac{\partial}{\partial x} \frac{1}{\epsilon} \frac{\phi^{\prime}\left(\frac{x-a}{\epsilon}\right)}{\phi\left(\frac{x-a}{\epsilon}\right)}<0, \text { for }(x, y) \in \operatorname{supp}(G)
$$

Therefore, we conclude that

$$
\frac{\partial}{\partial x} \frac{\frac{\partial}{\partial x} G(x, y)}{G(x, y)}<0, \text { for }(x, y) \in \operatorname{supp}(G)
$$


As in the previous case, since $x \rightarrow G\left(x, y_{0}\right)$ is positive in the interior of its support $\operatorname{supp}(G)=\left[a_{1}, a_{2}\right] \times\left[\alpha_{2}, \beta_{2}\right]$, for each fixed $y_{0} \in\left(\alpha_{2}, \beta_{2}\right)$ there exists $x \in\left(a_{1}, a_{2}\right)$ such that $\frac{\partial}{\partial x} G\left(x, y_{0}\right)=0$. Therefore for each fixed $y_{0} \in\left(\alpha_{2}, \beta_{2}\right)$, there is a unique $x=c\left(y_{0}\right) \in\left(a_{1}, a_{2}\right)$, such that $\frac{\frac{\partial}{\partial x} G(x, y)}{G(x, y)}=0$. Moreover, since the function $\frac{\frac{\partial}{\partial x} G(x, y)}{G(x, y)}$ is independent of $y$ for $(x, y) \in \operatorname{supp}(G)$, we conclude that the function $y \mapsto c(y)$ is constant on $\left(\alpha_{2}, \beta_{2}\right)$. This completes the proof of lemma 3.12 .

Lemma 3.13. In the same setting as in Lemma 3.12, for any open neighborhood $V$ of $U=\operatorname{supp}(G)=\left[a_{1}, a_{2}\right] \times\left[\alpha_{2}, \beta_{2}\right]$, there exists a compactly supported diffeomorphism $\Phi: V \rightarrow V$, such that $H=\Phi^{*} G$ satisfies $\left|\frac{\partial}{\partial x} H\right| \leqslant \frac{3\|G\|_{\infty}}{a_{2}-a_{1}}$, and $\operatorname{supp}(H)=\operatorname{supp}(G)$.

Proof of Lemma 3.13. We divide the proof of the lemma into two steps:

Step I: Let $V$ be an open neighborhood of $U=\operatorname{supp}(G)=\left[a_{1}, a_{2}\right] \times\left[\alpha_{2}, \beta_{2}\right]$. Take $\widetilde{\alpha}_{2}<\alpha_{2}<\beta_{2}<\widetilde{\beta}_{2}$, such that $\left[a_{1}, a_{2}\right] \times\left[\widetilde{\alpha}_{2}, \widetilde{\beta}_{2}\right] \subset V$. Moreover, take $\widetilde{a}_{1}, \widetilde{a}_{2}$ such that

$$
a_{1}<\widetilde{a}_{1}<\min _{\left[\alpha_{2}, \beta_{2}\right]} c(y) \leq \max _{\left[\alpha_{2}, \beta_{2}\right]} c(y)<\widetilde{a}_{2}<a_{2}
$$

and,

$$
\widetilde{a}_{1}<\frac{a_{1}+a_{2}}{2}<\widetilde{a}_{2}
$$

One can easily find a smooth family of diffeomorphisms $f^{t}:\left(a_{1}, a_{2}\right) \rightarrow\left(a_{1}, a_{2}\right)$, $t \in\left(\widetilde{a}_{1}, \widetilde{a}_{2}\right)$, such that:

$$
\left\{\begin{array}{l}
\operatorname{supp}\left(f^{t}\right) \subset\left[\widetilde{a}_{1}, \widetilde{a}_{2}\right] \\
f^{t}\left(\frac{a_{1}+a_{2}}{2}\right)=t \\
f^{\frac{a_{1}+a_{2}}{2}}=\mathbb{1}_{\left(a_{1}, a_{2}\right)}
\end{array}\right.
$$

We extend the function $c(y)$ to a smooth function on the interval $\left(\widetilde{\alpha}_{2}, \widetilde{\beta}_{2}\right)$, such that $c(y)=\frac{a_{1}+a_{2}}{2}$, for $y$ close enough to the points $\widetilde{\alpha}_{2}, \widetilde{\beta}_{2}$. Next, define a diffeomorphism

$$
\Psi_{1}:\left(a_{1}, a_{2}\right) \times\left(\widetilde{\alpha}_{2}, \widetilde{\beta}_{2}\right) \rightarrow\left(a_{1}, a_{2}\right) \times\left(\widetilde{\alpha}_{2}, \widetilde{\beta}_{2}\right)
$$

by the requirement:

$$
\Psi_{1}(x, y)=\left(f^{c(y)}(x), y\right)
$$

It is not hard to check that the diffeomorphism $\Psi_{1}$ is the identity near the boundary of the rectangle $\left(a_{1}, a_{2}\right) \times\left(\widetilde{\alpha}_{2}, \widetilde{\beta}_{2}\right)$, and therefore one can extend it by the identity, allowing ourselves a slight abuse of notation, to a diffeomorphism $\Psi_{1}: V \rightarrow V$. Denote $G_{1}=\Psi_{1}^{*} G$. It follows from the definition of $\Psi_{1}$ that for $y \in\left(\alpha_{2}, \beta_{2}\right)$, one has:

$$
\begin{cases}\frac{\partial}{\partial x} G_{1}(x, y)>0, & \text { for } a_{1}<x<\frac{a_{1}+a_{2}}{2}, \\ \frac{\partial}{\partial x} G_{1}(x, y)<0, & \text { for } \frac{a_{1}+a_{2}}{2}<x<a_{2}\end{cases}
$$


and moreover that $\operatorname{supp}\left(G_{1}\right)=\left[a_{1}, a_{2}\right] \times\left[\alpha_{2}, \beta_{2}\right]$, and $G_{1}(x, y)=u_{1}(x) v_{1}(y)$ for $x \in$ $\left[a_{1}, a_{2}\right]$ and $y$ being near $\alpha_{2}$ or $\beta_{2}$, where $u_{1}(x)=\left(f^{c\left(\alpha_{2}\right)}\right)^{*}\left(u(x) \phi\left(\frac{x-a}{\epsilon}\right)\right), v_{1}(x)=v(x)$.

\section{Step II:}

Let $0<\epsilon<\frac{a_{2}-a_{1}}{10}$, and consider three families of smooth positive functions $\chi_{j}^{\epsilon}$ : $\left[a_{1}, a_{2}\right] \rightarrow[0,1]$, where $j=1,2,3$, such that the following holds:

$$
\begin{aligned}
& \chi_{1}^{\epsilon}(x)= \begin{cases}1, & \text { for } x \in\left[a_{1}, a_{1}+\epsilon\right] \cup\left[\frac{a_{1}+a_{2}}{2}-\epsilon, \frac{a_{1}+a_{2}}{2}+\epsilon\right] \cup\left[a_{2}-\epsilon, a_{2}\right], \\
0, & \text { for } x \in\left[a_{1}+2 \epsilon, \frac{a_{1}+a_{2}}{2}-2 \epsilon\right] \cup\left[\frac{a_{1}+a_{2}}{2}+2 \epsilon, a_{2}-2 \epsilon\right],\end{cases} \\
& \chi_{2}^{\epsilon}(x)= \begin{cases}0, & \text { for } x \in\left[a_{1}, a_{1}+\epsilon\right] \cup\left[\frac{a_{1}+a_{2}}{2}-\epsilon, a_{2}\right], \\
1, & \text { for } x \in\left[a_{1}+2 \epsilon, \frac{a_{1}+a_{2}}{2}-2 \epsilon\right],\end{cases} \\
& \chi_{3}^{\epsilon}(x)= \begin{cases}0, & \text { for } x \in\left[a_{1}, \frac{a_{1}+a_{2}}{2}+\epsilon\right] \cup\left[a_{2}-\epsilon, a_{2}\right], \\
1, & \text { for } x \in\left[\frac{a_{1}+a_{2}}{2}+2 \epsilon, a_{2}-2 \epsilon\right],\end{cases}
\end{aligned}
$$

and moreover,

$$
\begin{cases}\chi_{2}^{\epsilon}(x)>0, & \text { for } x \in\left(a_{1}+\epsilon, \frac{a_{1}+a_{2}}{2}-\epsilon\right), \\ \chi_{3}^{\epsilon}(x)>0, & \text { for } x \in\left(\frac{a_{1}+a_{2}}{2}+\epsilon, a_{2}-\epsilon\right)\end{cases}
$$

Next, denote by $C_{0}^{\infty}\left(\left[a_{1}, a_{2}\right]\right)$ the set of smooth functions $\left[a_{1}, a_{2}\right] \rightarrow \mathbb{R}$, such that the derivatives of any order (including zero) vanish at the boundary points $a_{1}$ and $a_{2}$. Fix $g \in C_{0}^{\infty}\left(\left[a_{1}, a_{2}\right]\right)$, and define $h_{\epsilon}(x)$ by:

$$
h_{\epsilon}(x)=g^{\prime}(x) \chi_{1}^{\epsilon}(x)+A \chi_{2}^{\epsilon}(x)-B \chi_{3}^{\epsilon}(x),
$$

where $A$ and $B$ are two constants given by:

$$
A=\frac{g\left(\frac{a_{1}+a_{2}}{2}\right)-\int_{a_{1}}^{\frac{a_{1}+a_{2}}{2}} g^{\prime}(x) \chi_{1}^{\epsilon}(x) d x}{\int_{a_{1}}^{\frac{a_{1}+a_{2}}{2}} \chi_{2}^{\epsilon}(x) d x},
$$

and

$$
B=\frac{g\left(\frac{a_{1}+a_{2}}{2}\right)+\int_{\frac{a_{1}+a_{2}}{2}}^{a_{2}} g^{\prime}(x) \chi_{1}^{\epsilon}(x) d x}{\int_{\frac{a_{1}+a_{2}}{2}}^{a_{2}} \chi_{3}^{\epsilon}(x) d x} .
$$

Note that one has:

$$
\int_{a_{1}}^{\frac{a_{1}+a_{2}}{2}} h_{\epsilon}(x) d x=g\left(\frac{a_{1}+a_{2}}{2}\right),
$$

and

$$
\int_{\frac{a_{1}+a_{2}}{2}}^{a_{2}} h_{\epsilon}(x) d x=-g\left(\frac{a_{1}+a_{2}}{2}\right) .
$$


Let $g_{\epsilon}:\left[a_{1}, a_{2}\right] \rightarrow \mathbb{R}$ be the unique function such that $g_{\epsilon}^{\prime}(x)=h_{\epsilon}(x)$, and $g_{\epsilon}\left(a_{1}\right)=0$. It follows from the definition that

$$
g_{\epsilon}(x)=g(x), \text { for } x \in\left[a_{1}, a_{1}+\epsilon\right] \cup\left[\frac{a_{1}+a_{2}}{2}-\epsilon, \frac{a_{1}+a_{2}}{2}+\epsilon\right] \cup\left[a_{2}-\epsilon, a_{2}\right],
$$

and in particular, $g_{\epsilon} \in C_{0}^{\infty}\left(\left[a_{1}, a_{2}\right]\right)$. Note moreover that if $g(x)$ satisfies $g^{\prime}(x)>0$ for $x \in\left(a_{1}, \frac{a_{1}+a_{2}}{2}\right)$ and $g^{\prime}(x)<0$ for $x \in\left(\frac{a_{1}+a_{2}}{2}, a_{2}\right)$, then so is $g_{\epsilon}(x)$ i.e., $g_{\epsilon}^{\prime}(x)>0$ for $x \in\left(a_{1}, \frac{a_{1}+a_{2}}{2}\right)$ and $g_{\epsilon}^{\prime}(x)<0$ for $x \in\left(\frac{a_{1}+a_{2}}{2}, a_{2}\right)$.

Next, we define a family of operators $L_{\epsilon}: C_{0}^{\infty}\left(\left[a_{1}, a_{2}\right]\right) \rightarrow C_{0}^{\infty}\left(\left[a_{1}, a_{2}\right]\right)$, by the requirement that $L_{\epsilon} g=g_{\epsilon}$. It is not hard to check that $L_{\epsilon}$ is linear, and continuous in the $C^{\infty}$-topology. Moreover, let

$$
\mathcal{I}_{\epsilon}:=\left[a_{1}, a_{1}+2 \epsilon\right] \cup\left[\frac{a_{1}+a_{2}}{2}-2 \epsilon, \frac{a_{1}+a_{2}}{2}+2 \epsilon\right] \cup\left[a_{2}-2 \epsilon, a_{2}\right]
$$

Then, from the definition of $g_{\epsilon}$, and the fact that $\chi_{2}^{\epsilon}$ and $\chi_{3}^{\epsilon}$ has disjoint support, one has the following estimate:

$$
\max _{\left[a_{1}, a_{2}\right]}\left|g_{\epsilon}^{\prime}(x)\right| \leqslant \max _{x \in \mathcal{I}_{\epsilon}}\left|g^{\prime}(x)\right|+\max \{|A|,|B|\}
$$

Furthermore, from the definition of $A$ and $B$ one has:

$$
|A|,|B| \leqslant \frac{\left|g\left(\frac{a_{1}+a_{2}}{2}\right)\right|+4 \epsilon \max _{x \in \mathcal{I}_{\epsilon}}\left|g^{\prime}(x)\right|}{\frac{a_{2}-a_{1}}{2}-4 \epsilon} .
$$

Therefore, we conclude that

$$
\max _{\left[a_{1}, a_{2}\right]}\left|g_{\epsilon}^{\prime}(x)\right| \leqslant \frac{\left|g\left(\frac{a_{1}+a_{2}}{2}\right)\right|}{\frac{a_{2}-a_{1}}{2}-4 \epsilon}+\left(1+\frac{4 \epsilon}{\frac{a_{2}-a_{1}}{2}-4 \epsilon}\right) \max _{x \in \mathcal{I}_{\epsilon}}\left|g^{\prime}(x)\right| .
$$

Next, define $H_{\epsilon}:\left[a_{1}, a_{2}\right] \times\left[\alpha_{2}, \beta_{2}\right] \rightarrow \mathbb{R}$ by $H_{\epsilon}(\cdot, y)=L_{\epsilon} G_{1}(\cdot, y)$ for every $y \in\left[\alpha_{2}, \beta_{2}\right]$. Note that $\left.H_{\epsilon}\right|_{\left[a_{1}, a_{2}\right] \times\left[\alpha_{2}, \beta_{2}\right]}$ is a smooth function. Moreover, if $\epsilon>0$ is small enough, then from 3.1 .26 we conclude that

$$
\left|\frac{\partial}{\partial x} H_{\epsilon}(x, y)\right| \leqslant \frac{3\left\|G_{1}\right\|_{\infty}}{a_{2}-a_{1}}=\frac{3\|G\|_{\infty}}{a_{2}-a_{1}}, \text { for every }(x, y) \in\left[a_{1}, a_{2}\right] \times\left[\alpha_{2}, \beta_{2}\right]
$$

We fix such an $\epsilon$, and set $H:=H_{\epsilon}$. From the definition of $H$ and (3.1.25 one has:

$$
\begin{cases}\frac{\partial}{\partial x} H(x, y)>0, & \text { for } a_{1}<x<\frac{a_{1}+a_{2}}{2} \\ \frac{\partial}{\partial x} H(x, y)<0, & \text { for } \frac{a_{1}+a_{2}}{2}<x<a_{2}\end{cases}
$$

for any $y \in\left(\alpha_{2}, \beta_{2}\right)$. Furthermore,

$$
H(x, y)=G_{1}(x, y)
$$


for $x \in\left[a_{1}, a_{1}+\epsilon\right] \cup\left[\frac{a_{1}+a_{2}}{2}-\epsilon, \frac{a_{1}+a_{2}}{2}+\epsilon\right] \cup\left[a_{2}-\epsilon, a_{2}\right]$, and $y \in\left(\alpha_{2}, \beta_{2}\right)$. Note moreover that since the operator $L_{\epsilon}$ is linear, one has that $H(x, y)=\left(L_{\epsilon} u_{1}\right)(x) v_{1}(y)$ for any $x \in\left[a_{1}, a_{2}\right]$ and $y$ being near the boundary points $\alpha_{2}$ or $\beta_{2}$.

It follows from (3.1.27) and (3.1.28 above, that there is a unique diffeomorphism $\Psi_{2}:\left(a_{1}, a_{2}\right) \times\left(\alpha_{2}, \beta_{2}\right) \rightarrow\left(a_{1}, a_{2}\right) \times\left(\alpha_{2}, \beta_{2}\right)$, of the form $\Psi_{2}(x, y)=(w(x, y), y)$, such that

$$
\left.H\right|_{\left(a_{1}, a_{2}\right) \times\left(\alpha_{2}, \beta_{2}\right)}=\left.\Psi_{2}^{*} G_{1}\right|_{\left(a_{1}, a_{2}\right) \times\left(\alpha_{2}, \beta_{2}\right)},
$$

and $\operatorname{supp}(\Psi) \subset\left(\left[a_{1}+\epsilon, \frac{a_{1}+a_{2}}{2}-\epsilon\right] \cup\left[\frac{a_{1}+a_{2}}{2}+\epsilon, a_{2}-\epsilon\right]\right) \times\left[\alpha_{2}, \beta_{2}\right]$. Moreover, we have $G_{1}(x, y)=u_{1}(x) v_{1}(y), H(x, y)=\left(L_{\epsilon} u_{1}\right)(x) v_{1}(y)$ for $x \in\left[a_{1}, a_{2}\right]$ and $y$ being near $\alpha_{2}$ or $\beta_{2}$. From this we conclude that $w(x, y)$ is independent of $y$, for $y$ being close to $\alpha_{2}, \beta_{2}$. From Step I, we have $\widetilde{\alpha}_{2}<\alpha_{2}<\beta_{2}<\widetilde{\beta}_{2}$, such that $\left[a_{1}, a_{2}\right] \times\left[\widetilde{\alpha}_{2}, \widetilde{\beta}_{2}\right] \subset V$. One can easily extend the diffeomorphism $\Psi_{2}$ to

$$
\Psi_{2}:\left(a_{1}, a_{2}\right) \times\left(\widetilde{\alpha}_{2}, \widetilde{\beta}_{2}\right) \rightarrow\left(a_{1}, a_{2}\right) \times\left(\widetilde{\alpha}_{2}, \widetilde{\beta}_{2}\right)
$$

such that $\Psi_{2}$ is the identity diffeomorphism near the boundary of $\left(a_{1}, a_{2}\right) \times\left(\widetilde{\alpha}_{2}, \widetilde{\beta}_{2}\right)$. Then we can extend $\Psi_{2}$ by the identity to be a diffeomorphism $\Psi_{2}: V \rightarrow V$. We have $H=\Psi_{2}^{*} G_{1}$.

Finally, denote $\Phi=\Psi_{1} \Psi_{2}: V \rightarrow V$. The diffeomorphism $\Phi$ is compactly supported inside $V$, and $H=\Phi^{*} G$ satisfies

$$
\left|\frac{\partial}{\partial x} H\right| \leqslant \frac{3\|G\|_{\infty}}{a_{2}-a_{1}}, \text { and } \operatorname{supp}(H)=\operatorname{supp}(G)
$$

This completes the proof of the lemma.

We are finally in a position to prove Lemma 3.8.

Proof of Lemma 3.8. Let $f: \mathbb{R}^{2} \rightarrow \mathbb{R}$ be a smooth function with $\|f\|_{\infty} \leqslant 1$, and $\operatorname{supp}(f) \subset \operatorname{int}(R)$. We fix some parameters $\alpha_{i}, \alpha_{i}^{\prime}, \beta_{i}, \beta_{i}^{\prime}$, where $i=1,2$, such that $0<\alpha_{i}<\alpha_{i}^{\prime}<\beta_{i}^{\prime}<\beta_{i}<L_{i}$, for $i=1,2 ; \beta_{1}-\alpha_{1}>\frac{3}{4} L_{1} ;$ and

$$
\operatorname{supp}(f) \subset \operatorname{int}\left(\left[\alpha_{1}^{\prime}, \beta_{1}^{\prime}\right] \times\left[\alpha_{2}^{\prime}, \beta_{2}^{\prime}\right]\right) \subset \operatorname{int}\left(\left[\alpha_{1}, \beta_{1}\right] \times\left[\alpha_{2}, \beta_{2}\right]\right) \subset \operatorname{int}(R)
$$

Moreover, we choose a smooth function $u:\left[0, L_{1}\right] \rightarrow \mathbb{R}$, such that $u(x)=e^{\frac{-1}{x-\alpha_{1}}}$ near $\alpha_{1}, u(x)=e^{\frac{-1}{\beta_{1}-x}}$ near $\beta_{1}, u(x)=1$ on $\left[\alpha_{1}^{\prime}, \beta_{1}^{\prime}\right]$, and $\|u\|_{\infty}=1$. Similarly, we take $v:\left[0, L_{2}\right] \rightarrow \mathbb{R}$, with $v(y)=e^{\frac{-1}{y-\alpha_{2}}}$ near $\alpha_{2}, v(y)=e^{\frac{-1}{\beta_{2}-y}}$ near $\beta_{2}, v(y)=2$ on $\left[\alpha_{2}^{\prime}, \beta_{2}^{\prime}\right]$, and $\|v\|_{\infty}=2$. Next, we consider the decomposition $f=F_{1}-F_{2}$, where

$$
F_{1}(x, y)=f(x, y)+u(x) v(y), \text { and } F_{2}(x, y)=u(x) v(y)
$$

We have $\left\|F_{\varsigma}(x, y)\right\|_{\infty} \leqslant 3$ for $\varsigma \in\{1,2\}$. From Lemma 3.13 it follows that there is $\epsilon_{0}>0$ such that for any $0<\epsilon<\epsilon_{0}$, and any $a \in \mathbb{R}$, the following holds: let $G_{\varsigma}(x, y)=$ 
$F_{\varsigma}(x, y) \phi\left(\frac{x-a}{\epsilon}\right)$, where $\varsigma \in\{1,2\}$ (we may and shall assume in what follows that $\left.G_{\varsigma} \neq 0\right)$. Take $V^{\varsigma}$ to be any open neighborhood of $U^{\varsigma}:=\operatorname{supp}\left(G_{\varsigma}\right)=\left[a_{1}^{\varsigma}, a_{2}^{\varsigma}\right] \times\left[\alpha_{2}, \beta_{2}\right]$. Then, there is a compactly supported diffeomorphism $\Phi^{\varsigma}: V^{\varsigma} \rightarrow V^{\varsigma}$, such that $H_{\varsigma}=\left(\Phi^{\varsigma}\right)^{*} G_{\varsigma}$ satisfies

$$
\left|\frac{\partial}{\partial x} H_{\varsigma}\right| \leqslant \frac{9}{a_{2}^{\varsigma}-a_{1}^{\varsigma}} \text {, and } \operatorname{supp}\left(H_{\varsigma}\right)=\operatorname{supp}\left(G_{\varsigma}\right)
$$

Fix $0<\epsilon<\epsilon_{0}$ as above. For $n \in \mathbb{Z}$ and $\varsigma \in\{1,2\}$ denote $G_{\varsigma, n}=F_{\varsigma}(x, y) \phi\left(\frac{x-n \epsilon}{\epsilon}\right)$. Note that $F_{\varsigma}=\sum_{n \in \mathbb{Z}} G_{\varsigma, n}$, and that only finitely many summands are not identically zero. For $i=1,2,3,4$, let $K_{\varsigma, i}=\sum_{j \in \mathbb{Z}} G_{\varsigma, i+4 j}$. Note moreover that the supports of all the non-zero summands of $K_{\varsigma, i}$ are pairwise disjoint, and $F_{\varsigma}=\sum_{i=1}^{4} K_{\varsigma, i}$, and thus $f=$ $\sum_{\varsigma=1}^{2} \sum_{i=1}^{4} K_{\varsigma, i}$. Next, we fix $1 \leqslant i_{0} \leqslant 4$. Consider $K_{\varsigma, i_{0}}=\sum_{j \in \mathbb{Z}} G_{\varsigma, i_{0}+4 j}$, and choose pairwise disjoint open neighborhoods $V_{i_{0}, j}^{\varsigma} \supset \operatorname{supp}\left(G_{\varsigma, i_{0}+4 j}\right)$ of those summands which are not identically zero. Now, apply Lemma 3.13 to each element in the decomposition $K_{\varsigma, i_{0}}=\sum_{j \in \mathbb{Z}} G_{\varsigma, i_{0}+4 j}$. We obtain that for any non-zero summand $G_{\varsigma, i_{0}+4 j}$, there is a compactly supported diffeomorphism $\Phi_{i_{0}, j}^{\varsigma}: V_{i_{0}, j}^{\varsigma} \rightarrow V_{i_{0}, j}^{\varsigma}$, such that the function $H_{i_{0}, j}^{\varsigma}=\left(\Phi_{i_{0}, j}^{\varsigma}\right)^{*} G_{\varsigma, i_{0}+4 j}$ satisfies

$$
\left|\frac{\partial}{\partial x} H_{i_{0}, j}^{\varsigma}\right| \leqslant \frac{9}{\mu\left(\pi_{x}\left(\operatorname{supp}\left(G_{\varsigma, i_{0}+4 j}\right)\right)\right)}, \text { and } \operatorname{supp}\left(H_{i_{0}, j}^{\varsigma}\right)=\operatorname{supp}\left(G_{\varsigma, i_{0}+4 j}\right)
$$

Here $\pi_{x}$ denotes the projection to the interval $\left[0, L_{1}\right]$, and $\mu$ is the Lebesgue measure. Note that the supports $\left\{\operatorname{supp}\left(\Phi_{i_{0}, j}^{\varsigma}\right)\right\}$ are mutually disjoint. We shall denote by $\widetilde{\Phi}_{i_{0}}^{\varsigma}$ the composition of all the $\Phi_{i_{0}, j}^{\varsigma}$ 's for which $G_{\varsigma, i_{0}+4 j} \neq 0$. Moreover, we denote by $\Pi_{i_{0}, k}^{\varsigma}$, $k=1,2, \ldots, M_{i_{0}}$ all the non-empty supports among $\left\{\operatorname{supp}\left(G_{\varsigma, i_{0}+4 j}\right)\right\}$. Note that each $\Pi_{i_{0}, k}^{\varsigma}$ is a rectangle contained in $\left[\alpha_{1}, \beta_{1}\right] \times\left[\alpha_{2}, \beta_{2}\right]$. Consider a sequence of rectangles

$$
\widetilde{\Pi}_{i_{0}, k}^{\varsigma}:=\left[\alpha_{1}, \beta_{1}\right] \times\left[\alpha_{2}+(2 k-1) \frac{\beta_{2}-\alpha_{2}}{2 M_{i_{0}}}, \alpha_{2}+2 k \frac{\beta_{2}-\alpha_{2}}{2 M_{i_{0}}}\right]
$$

It is not hard to check that there exists a diffeomorphism $\Psi_{i_{0}}^{\varsigma}: R \rightarrow R$, such that $\Psi_{i_{0}}^{\varsigma}\left(\widetilde{\Pi}_{i_{0}, k}^{\varsigma}\right)=\Pi_{i_{0}, k}^{\varsigma}$, and moreover that on each $\widetilde{\Pi}_{i_{0}, k}^{\varsigma}$ it coincides with a linear contraction on the directions of the axes, composed with a translation. As a result, for $k_{\varsigma, i_{0}}:=\left(\Psi_{i_{0}}^{\varsigma}\right)^{*}\left(\widetilde{\Phi}_{i_{0}}^{\varsigma}\right)^{*} K_{\varsigma, i_{0}}$, one has $\left|\frac{\partial}{\partial x} k_{\varsigma, i_{0}}\right| \leqslant \frac{9}{\beta_{1}-\alpha_{1}}<\frac{12}{L_{1}}$. The proof of Lemma 3.8 is now complete.

\subsection{Theorem 3.4 - the higher-dimensional case}

The proof of Theorem 3.4 for arbitrary dimension relies on the 2-dimensional case, and on the following proposition, the proof of which we postpone to Subsection 3.2.1.

Proposition 3.14. There is a finite family of functions $\mathcal{F} \subset C_{c}^{\infty}(W)$, such that: 
(i) Any $f \in C_{c}^{\infty}(W)$ that can be represented as a product $f(q, p)=\prod_{i=1}^{n} f_{i}\left(q_{i}, p_{i}\right)$, for some $f_{i} \in C_{c}^{\infty}\left(I^{2}\right)$, satisfies that $\|f\|_{\mathcal{F}, \max } \leqslant C\|f\|_{\infty}$, for some constant $C$.

(ii) For any $f \in C_{c}^{\infty}(W)$, one has $\|f\|_{\mathcal{F}, \max } \leqslant C\|f\|_{C^{2 n+1}}$, for some constant $C$.

Remark 3.15. In what follows, we fix $\mathcal{F}$ to be the collection of functions given by Proposition 3.14 above. Moreover, in order to simplify the presentation, we shall use $x_{1}=q_{1}, x_{2}=p_{1}, \ldots, x_{2 n-1}=q_{n}, x_{2 n}=p_{n}$, as another notation for the coordinates of a point $x=\left(q_{1}, p_{1}, \ldots, q_{2 n}, p_{2 n}\right)$ in the $2 n$-dimensional cube $W=(-L, L)^{2 n}$.

Proof of Theorem 3.4 (the higher dimensional case). For simplicity, the proof of the theorem is divided into two steps:

Step I (Decomposing the function): We consider a smooth function $r:[-1,1] \rightarrow \mathbb{R}$, satisfying:

$$
r(t)= \begin{cases}1 & \text { for } t \in\left[-\frac{1}{3}, \frac{1}{3}\right], \\ 0 & \text { for } t \in\left[-1,-\frac{2}{3}\right] \cup\left[\frac{2}{3}, 1\right],\end{cases}
$$

and such that $\sum_{i \in \mathbb{Z}} r(t+i)=1$, and $\|r\|_{\infty}=1$. For any $\epsilon>0$, we denote

$$
\mathcal{R}^{\epsilon}(x)=\mathcal{R}^{\epsilon}\left(x_{1}, x_{2}, \ldots, x_{2 n}\right)=\prod_{i=1}^{2 n} r\left(\frac{x_{i}}{\epsilon}\right)
$$

Clearly, one has $\sum_{v \in \epsilon \mathbb{Z}^{2 n}} \mathcal{R}^{\epsilon}(x-v)=\mathbb{1}(x)$. Moreover, for a sufficiently small $\epsilon>0$, and a point $w \in \mathfrak{X}:=\{0,1,2,3\}^{2 n}$, we consider a finite grid $\Gamma_{w}^{\epsilon} \subset W$ given by

$$
\Gamma_{w}^{\epsilon}=\epsilon w+4 \epsilon \mathbb{Z}^{2 n} \cap(-L+3 \epsilon, L-3 \epsilon)^{2 n}
$$

Furthermore, we define a partition function $\mathcal{R}_{w}^{\epsilon}(x)$ by:

$$
\mathcal{R}_{w}^{\epsilon}(x)=\sum_{v \in \Gamma_{w}^{\epsilon}} \mathcal{R}^{\epsilon}(x-v)
$$

Note that $\sum_{w \in \mathcal{X}} \mathcal{R}_{w}^{\epsilon}(x)=\mathbb{1}(x)$ for any $x \in(-L+4 \epsilon, L-4 \epsilon)^{2 n}$. Next, consider an arbitrary function $f \in C_{c}^{\infty}(W)$. Take $\epsilon_{0}>0$ with $\operatorname{supp}(f) \subset\left(-L+4 \epsilon_{0}, L-4 \epsilon_{0}\right)^{2 n}$, and fix $\epsilon<\epsilon_{0}$. For any $w \in \mathfrak{X}$, denote $f_{w}(x)=\mathcal{R}_{w}^{\epsilon}(x) f(x)$. Note that

$$
f(x)=\sum_{w \in \mathfrak{X}} f_{w}(x)
$$

Moreover, for a fix $w \in \mathfrak{X}$ one has

$$
f_{w}(x)=\sum_{v \in \Gamma_{w}^{\epsilon}} \mathcal{R}^{\epsilon}(x-v) f(x),
$$

where the support of each summand satisfies

$$
\operatorname{supp}\left(\mathcal{R}^{\epsilon}(x-v) f(x)\right) \subset v+\left[-\frac{2 \epsilon}{3}, \frac{2 \epsilon}{3}\right]^{2 n}, \text { for } v \in \Gamma_{w}^{\epsilon}
$$


Step II (Estimating the norm $\|f\|_{\mathcal{F}, \text { max }}$ ): Fix $v \in \Gamma_{w}^{\epsilon}$, and consider the decomposition of $f \in C_{c}^{\infty}(W)$ to a Taylor polynomial of order $2 n+1$ and a remainder, around the point $v$ :

$$
f(x)=P_{2 n+1}^{v}(x-v)+R_{2 n+1}^{v}(x-v)
$$

It follows from (3.2.32) above that $f_{w}(x)=g_{w}(x)+h_{w}(x)$, where

$$
g_{w}(x)=\sum_{v \in \Gamma_{w}^{\epsilon}} \mathcal{R}^{\epsilon}(x-v) P_{2 n+1}^{v}(x-v), \text { and } h_{w}(x)=\sum_{v \in \Gamma_{w}^{\epsilon}} \mathcal{R}^{\epsilon}(x-v) R_{2 n+1}^{v}(x-v)
$$

Lemma 3.16. With the above notations, there is a constant $C=C(n)$ such that

$$
\left\|h_{w}\right\|_{C^{2 n+1}} \leqslant C \epsilon\|f\|_{C^{2 n+2}}
$$

Proof of Lemma 3.16. From the fact that the family $\left\{\mathcal{R}^{\epsilon}(x-v) R_{2 n+1}^{v}(x-v)\right\}_{v \in \Gamma_{w}^{\epsilon}}$ has mutually disjoint support, and the definition of the norm $\|\cdot\|_{C^{2 n+1}}$, it follows that there is a constant $C$ (depending on the dimension) such that

$$
\begin{aligned}
\left\|h_{w}(x)\right\|_{C^{2 n+1}} & \leq \max _{v \in \Gamma_{w}^{\epsilon}}\left\|\mathcal{R}^{\epsilon}(x-v) R_{2 n+1}^{v}(x-v)\right\|_{C^{2 n+1}} \\
& \leq C(n) \max _{v \in \Gamma_{w}^{\epsilon}}\left(\max _{0 \leq k \leq 2 n+1}\left\|\mathcal{R}^{\epsilon}(x-v)\right\|_{C^{k}}\left\|R_{2 n+1}^{v}(x-v)\right\|_{C^{2 n+1-k}}\right)
\end{aligned}
$$

Note that from the definition of $\mathcal{R}^{\epsilon}$ it follows that for every $0 \leq k \leq 2 n+1$, one has

$$
\left\|\mathcal{R}^{\epsilon}(x-v)\right\|_{C^{k}} \leqslant C^{\prime} \epsilon^{-k},
$$

for some constant $C^{\prime}$ (independent of $k$ ). Note moreover, that for $0 \leq k \leq 2 n+1$,

$$
\left\|R_{2 n+1}^{v}(x-v)\right\|_{C^{2 n+1-k}} \leqslant C^{\prime \prime}\|f\|_{C^{2 n+2}} \epsilon^{1+k},
$$

for some constant $C^{\prime \prime}$. Indeed, let $\alpha$ be a multiindex with $|\alpha|=2 n+1-k$, and consider the order- $k$ Taylor's expension of $\partial^{\alpha} f$ near the point $v$. The remainder equals to $\partial^{\alpha} R_{2 n+1}^{v}(x-v)$, and the estimate (3.2.33) follows from the standard bound on the size of the remainder. This completes the proof of the lemma.

Corollary 3.17. From Proposition 3.14 (ii), and Lemma 3.16, we conclude that:

$$
\left\|h_{w}\right\|_{\mathcal{F}, \max } \leqslant C \epsilon\|f\|_{C^{2 n+2}}, \text { for some constant } C=C(n)
$$

To complete the proof of the theorem we shall need the following proposition:

Proposition 3.18. There is a constant $C=C(n)$ such that

$$
\left\|g_{w}\right\|_{\mathcal{F}, \max } \leqslant C\left(\sum_{i=0}^{2 n+1}\|f\|_{C^{i}} \epsilon^{i}\right)
$$

Postponing the proof of Proposition 3.18 to Subsection 3.2.2, we first complete the proof of Theorem 3.4. From (3.2.34) and (3.2.35), letting $\epsilon \rightarrow 0$, we conclude that

$$
\|f\|_{\mathcal{F}, \max } \leq C\|f\|_{\infty}
$$

for some absolute constant $C$, and the proof is complete. 


\subsubsection{Proof of Proposition 3.14}

Part (i): Let $W=\prod_{i=1}^{n} W_{i}^{2}$, where $W_{i}^{2}=(-L, L)^{2} \subset \mathbb{R}^{2}\left(q_{i}, p_{i}\right)$, and denote by $\mathcal{F}^{2}=\left\{\mathfrak{f}_{0}, \mathfrak{f}_{1}, \mathfrak{f}_{2}\right\}$ the collection of functions constructed in the proof of Theorem 3.4 in the 2-dimensional case. For any multi-index $\beta=\left(l_{1}, \ldots, l_{n}\right) \in \mathfrak{X}^{\prime}:=\{0,1,2\}^{n}$, we set $\mathfrak{f}_{\beta}(q, p)=\prod_{k=1}^{n} \mathfrak{f}_{l_{k}}\left(q_{k}, p_{k}\right)$. In what follows we denote by $\mathcal{F}$ the set $\left\{\mathfrak{f}_{\beta} ; \beta \in \mathfrak{X}^{\prime}\right\}$.

Consider $f \in C_{c}^{\infty}\left(W^{2 n}\right)$ of the form $f(q, p)=\prod_{i=1}^{n} f_{i}\left(q_{i}, p_{i}\right)$, where $f_{i} \in C_{c}^{\infty}\left(W_{i}\right)$. Let $\epsilon>0$. From the proof of Theorem 3.4 in the 2-dimensional case it follows that there exists functions $f_{i, k} \in \mathcal{L}_{\mathcal{F}^{2}}, i=1,2, \ldots, n ; k \in \mathbb{N}$, such that $f_{i, k} \stackrel{k \rightarrow \infty}{\longrightarrow} f_{i}$ in the $C^{\infty}$-topology, and such that $\left\|f_{i, k}\right\|_{\mathcal{L}_{\mathcal{F} 2}}<\left\|f_{i}\right\|_{\mathcal{F}^{2}, \max }+\epsilon$. Next, for every $1 \leq i \leq n$ and $k \in \mathbb{N}$, we decompose

$$
f_{i, k}=\sum_{j, l} c_{i, k}^{j, l}\left(\Phi_{i, k}^{j, l}\right)^{*} \mathfrak{f}_{l}
$$

where $\Phi_{i, k}^{j, l} \in \operatorname{Ham}_{c}\left(W_{i}, \omega\right) ; l \in\{0,1,2\}$, and,

$$
\sum_{j, l}\left|c_{i, k}^{j, l}\right|<\left\|f_{i, k}\right\|_{\mathcal{L}_{\mathcal{F}^{2}}}+\epsilon
$$

Denote $f^{k}(q, p)=\prod_{i=1}^{n} f_{i, k}\left(q_{i}, p_{i}\right)$. Clearly, $f^{k} \stackrel{k \rightarrow \infty}{\longrightarrow} f \in C_{c}^{\infty}(W)$ in the $C^{\infty}$-topology. Moreover, from 3.2.36 it follows that

$$
f^{k}=\sum_{\substack{\beta=\left(l_{1}, \ldots, l_{n}\right) \\ \gamma=\left(j_{1}, \ldots, j_{n}\right)}} c_{k}^{\gamma, \beta}\left(\Phi_{k}^{\gamma, \beta}\right)^{*} \mathfrak{f}_{\beta}
$$

where

$$
c_{k}^{\gamma, \beta}=\prod_{i=1}^{n} c_{i, k}^{j_{i}, l_{i}}, \text { and } \Phi_{k}^{\gamma, \beta}\left(q_{1}, p_{1}, \ldots, q_{n}, p_{n}\right)=\left(\Phi_{1, k}^{j_{1}, l_{1}}\left(q_{1}, p_{1}\right), \ldots, \Phi_{n, k}^{j_{n}, l_{n}}\left(q_{n}, p_{n}\right)\right)
$$

This shows that $f^{k} \in \mathcal{L}_{\mathcal{F}}$, and moreover that

$$
\begin{aligned}
\left\|f^{k}\right\|_{\mathcal{L}_{\mathcal{F}}} & \leqslant \sum_{\substack{\beta=\left(l_{1}, \ldots, l_{n}\right) \\
\gamma=\left(j_{1}, \ldots, j_{n}\right)}}\left|c_{k}^{\gamma, \beta}\right|=\prod_{i=1}^{n}\left(\sum_{j_{i}, l_{i}}\left|c_{i, k}^{j_{i}, l_{i}}\right|\right)<\prod_{i=1}^{n}\left(\left\|f_{i, k}\right\|_{\mathcal{L}_{\mathcal{F}^{2}}}+\epsilon\right) \\
& \leqslant \prod_{i=1}^{n}\left(\left\|f_{i}\right\|_{\mathcal{F}^{2}, \max }+2 \epsilon\right)
\end{aligned}
$$

Recall, that from the proof of Theorem 3.4 in the 2-dimensional case one has

$$
\left\|f_{i}\right\|_{\mathcal{F}^{2}, \max } \leqslant C\left\|f_{i}\right\|_{\infty},
$$

for some absolute constant $C$. Combining this with (3.2.38) we conclude that

$$
\left\|f^{k}\right\|_{\mathcal{L}_{\mathcal{F}}} \leqslant \prod_{i=1}^{n}\left(C\left\|f_{i}\right\|_{\infty}+2 \epsilon\right)
$$


and therefore

$$
\|f\|_{\mathcal{F}, \text { max }} \leqslant \liminf _{k \rightarrow \infty}\left\|f^{k}\right\|_{\mathcal{L}_{\mathcal{F}}} \leqslant \prod_{i=1}^{n}\left(C\left\|f_{i}\right\|_{\infty}+2 \epsilon\right)
$$

In particular, for any $\epsilon>0$, one has

$$
\|f\|_{\mathcal{F}, \max } \leqslant \prod_{i=1}^{n}\left(C\left\|f_{i}\right\|_{\infty}+2 \epsilon\right)
$$

Taking $\epsilon \rightarrow 0$, we obtain

$$
\|f\|_{\mathcal{F}, \max } \leqslant C^{n} \prod_{i=1}^{n}\left\|f_{i}\right\|_{\infty}=C^{n}\|f\|_{\infty}
$$

This completes the proof of part (i) of Proposition 3.14.

For the proof of the second part of Proposition 3.14 we shall need the following preliminaries. Let $f$ be an integrable function on the $m$-dimensional torus $\mathbb{T}^{m}$, and denote its Fourier coefficients by

$$
\hat{f}_{r}=\frac{1}{(2 \pi)^{m}} \int_{\mathbb{T}^{m}} f(t) e^{i r \cdot t} d t
$$

where $r=\left(r_{1}, \ldots, r_{m}\right) \in \mathbb{Z}^{m}$, and $t=\left(t_{1}, \ldots, t_{m}\right) \in \mathbb{T}^{m}$. We denote the $j^{t h}$-partial sum of the Fourier series of $f$ by

$$
S_{j}(f, t)=\sum_{\max \left|r_{l}\right| \leq j} \hat{f}_{r} e^{i r \cdot t}
$$

The next lemma is a well known result in Fourier analysis.

Lemma 3.19. Let $f \in C^{\infty}\left(\mathbb{T}^{m}\right)$. Then $S_{j}(f) \stackrel{j \rightarrow \infty}{\longrightarrow} f$ in the $C^{\infty}$-topology and

$$
\sum_{r \in \mathbb{Z}^{m}}\left|\hat{f}_{r}\right| \leq A\|f\|_{C^{2 n+1}}
$$

for some universal constant $A$.

Proof of Lemma 3.19. The fact that $S_{j}(f) \stackrel{j \rightarrow \infty}{\longrightarrow} f$ in the $C^{\infty}$-topology follows, e.g., from Theorem 33.7 in Section 79 of [9], and the fact that $\partial^{\alpha} S_{j}(f)=S_{j}\left(\partial^{\alpha} f\right)$ for every multi-index $\alpha$ and $j \geq 0$. For the estimate (3.2.39), we use Lemma 9.5 in Section 79 of [9] to obtain the following upper bound for the Fourier coefficients:

$$
\left|\hat{f}_{r}\right| \leq A_{1} \frac{\|f\|_{C^{2 n+1}}}{\|r\|^{2 n+1}} \text { for all } r \neq 0
$$

for some constant $A_{1}$. From this we conclude that

$$
\sum_{r \in \mathbb{Z}^{m}}\left|\hat{f}_{r}\right| \leq A_{2}\|f\|_{C^{2 n+1}} \int_{S^{2 n-1}} \int_{1}^{\infty} \rho^{-2 n-1} \rho^{2 n-1} d \rho d \theta \leq A_{3}\|f\|_{C^{2 n+1}},
$$

where $A=A_{3}$ is a constant which depends solely on the dimension. 
Remark 3.20. We remark that Lemma 3.19 holds (with different constants) for any torus of the form $T^{m}=(\mathbb{R} / a \mathbb{Z})^{m}$, where $a>0$. Moreover, the lemma holds if instead of the basis $\left\{e^{\frac{2 \pi i}{a} r t}\right\}$, we choose the trigonometric basis consists of products of $\left\{\cos \left(\frac{2 \pi}{a} r_{i} t_{i}\right)\right\}$ or $\left\{\sin \left(\frac{2 \pi}{a} r_{i} t_{i}\right)\right\}$ for $i=1, \ldots, m$.

We now turn to complete the proof of the second part of Proposition 3.14;

Proposition 3.14, Part (ii): Let $f \in C_{c}^{\infty}(W)$. By gluing together the boundary of the cube $W$ in an appropriate way, we obtain a well defined smooth function on the torus $T^{2 n}=(\mathbb{R} / 2 L \mathbb{Z})^{2 n}$, which by abuse of notation we still denote by $f$. We apply Lemma 3.19 to the function $f$ (note the comment regarding the trigonometric basis in Remark (3.20). We order the trigonometric basis in Remark 3.20 by $\left\{e_{k}\right\}_{k=1}^{\infty}$. Note that each $e_{k}$ is a product function with $\left\|e_{k}\right\|_{\infty}=1$. Denoting the corresponding Fourier sums of $f$ by $S_{k}=\sum_{i=1}^{k} c_{i} e_{i}$. We have $S_{k} \rightarrow f$ in the $C^{\infty}$-topology and $\sum_{k=1}^{\infty}\left|c_{k}\right| \leqslant A\|f\|_{C^{2 n+1}}$ for some $A=A(n)$. We turn back to the situation where we consider $f$ defined on $W$. Take any smooth cutoff function $\rho: W \rightarrow \mathbb{R}$, which equals 1 on $\operatorname{supp}(f)$, equals 0 near the boundary $\partial W$, and which has $\|\rho\|_{\infty}=1$ (one can easily find such $\rho$, since $\operatorname{supp}(f) \subset W)$. Then we have $\rho S_{k}=\sum_{i=1}^{k} c_{i} \rho e_{i} \rightarrow \rho f=f$ in $C_{c}^{\infty}(W)$, in the $C^{\infty}$ topology as well. Moreover, the functions $\left\{\rho e_{k}\right\}$ are product functions with $\left\|\rho e_{k}\right\|_{\infty} \leqslant 1$. From part (i) or Proposition 3.14, and Lemma 3.19, it follows that for a suitable collection $\mathcal{F}$, one has

$$
\left\|S_{k}\right\|_{\mathcal{F}, \max } \leqslant \sum_{i=1}^{k}\left|c_{i}\right|\left\|\rho e_{i}\right\|_{\mathcal{F}, \max } \leqslant C \sum_{i=1}^{k}\left|c_{i}\right| \leqslant C A\|f\|_{C^{2 n+1}} .
$$

Hence, from Remark 3.2 we conclude that

$$
\|f\|_{\mathcal{F}, \max } \leqslant C A\|f\|_{C^{2 n+1}} .
$$

The proof of the second part of the proposition is now complete.

\subsubsection{Proof of Proposition 3.18}

For any multi-index $\alpha=\left(i_{1}, i_{2}, \ldots, i_{2 n}\right)$, where $|\alpha| \leqslant 2 n+1$, denote

$$
g_{w}^{\alpha}(x)=\sum_{v=\left(v_{1}, v_{2}, \ldots, v_{2 n}\right) \in \Gamma_{w}^{\epsilon}} \frac{1}{i_{1} ! i_{2} ! \ldots i_{2 n} !} \frac{\partial f^{|\alpha|}}{\partial x_{1}^{i_{1}} \partial x_{2}^{i_{2}} \ldots \partial x_{2 n}^{i_{2 n}}}(v)\left(\prod_{j=1}^{2 n}\left(x_{j}-v_{j}\right)^{i_{j}}\right) \mathcal{R}^{\epsilon}(x-v)
$$

Note that the function $g_{w}$ is the sum of $g_{w}^{\alpha}$, for $\alpha=\left(i_{1}, i_{2}, \ldots, i_{2 n}\right)$ with $|\alpha| \leqslant 2 n+1$. Note moreover that each summand of $g_{w}^{\alpha}$ is a constant multiple of the function

$$
\Xi_{\alpha}(x-v):=\left(\prod_{j=1}^{2 n}\left(x_{j}-v_{j}\right)^{i_{j}}\right) \mathcal{R}^{\epsilon}(x-v)
$$


where

$$
\Xi_{\alpha}(x)=x_{1}^{i_{1}} x_{2}^{i_{2}} \ldots x_{2 n}^{i_{2 n}} \mathcal{R}^{\epsilon}(x)=\prod_{l=1}^{n} q_{l}^{i_{2 l-1}} p_{l}^{i_{2 l}} r\left(\frac{q_{l}}{\epsilon}\right) r\left(\frac{p_{l}}{\epsilon}\right)
$$

We shall need the following lemma which will be proven in Subsection 3.2.3

Lemma 3.21. Let $\xi \in C_{c}^{\infty}\left((-\epsilon, \epsilon)^{2 n}\right)$ be a compactly supported smooth function which can be represented as a product $\xi=\prod_{j=1}^{n} \xi_{j}\left(q_{j}, p_{j}\right)$, where $\xi_{j} \in C_{c}^{\infty}\left((-\epsilon, \epsilon)^{2}\right)$. Then, for every function $H(x)=\sum_{v \in \Gamma_{w}^{\epsilon}} a_{v} \xi(x-v)$, where $a_{v}$ are real coefficients and $\Gamma_{w}^{\epsilon}$ is the grid defined in (3.2.31), one has

$$
\|H\|_{\mathcal{F}, \max } \leqslant C\|H\|_{\infty}, \text { for some absolute constant } C
$$

Applying Lemma 3.21, with $\xi=\Xi_{\alpha}$, to the function $H=g_{w}^{\alpha}$, we conclude that

$$
\begin{aligned}
\left\|g_{w}^{\alpha}\right\|_{\mathcal{F}, \max } & \leqslant C\left\|g_{w}^{\alpha}\right\|_{\infty} \leqslant \frac{C}{i_{1} ! i_{2} ! \ldots i_{2 n} !}\left\|\Xi_{\alpha}\right\|_{\infty} \max _{v \in \Gamma_{w}^{\epsilon}} \frac{\partial f^{|\alpha|}}{\partial x_{1}^{i_{1}} \partial x_{2}^{i_{2}} \ldots \partial x_{2 n}^{i_{2 n}}}(v) \\
& \leqslant C\left\|\Xi_{\alpha}\right\|_{\infty}\|f\|_{C^{|\alpha|}}
\end{aligned}
$$

Since $\|r\|_{\infty}=1$, and $\operatorname{supp}(r) \subset(-\epsilon, \epsilon)$, it follows that $\left\|\Xi_{\alpha}\right\|_{\infty} \leq \epsilon^{|\alpha|}$. Thus, we obtain

$$
\left\|g_{w}^{\alpha}\right\|_{\mathcal{F}, \max } \leqslant C \epsilon^{|\alpha|}\|f\|_{C^{|\alpha|}}
$$

and hence

$$
\left\|g_{w}\right\|_{\mathcal{F}, \text { max }} \leqslant \sum_{|\alpha| \leqslant 2 n+1} C \epsilon^{|\alpha|}\|f\|_{C^{|\alpha|}} \leqslant C^{\prime} \sum_{k=0}^{2 n+1} \epsilon^{k}\|f\|_{C^{k}}
$$

This completes the proof of Proposition 3.18 .

\subsubsection{Proof of Lemma 3.21}

Note first that the grid $\Gamma_{w}^{\epsilon}=\epsilon w+4 \epsilon \mathbb{Z}^{2 n} \cap(-L+3 \epsilon, L-3 \epsilon)^{2 n}$ admits a decomposition into the product $\Gamma_{w}^{\epsilon}=\prod_{i=1}^{n} \gamma_{i}$, where $\gamma_{i}=\gamma_{i}^{\epsilon, w} \subset(-L+3 \epsilon, L-3 \epsilon)^{2} \subset(-L, L)^{2}$ are grids on the plane. Next, let $H$ be as in Lemma 3.21. Given a bijection $\tau: \Gamma_{w}^{\epsilon} \rightarrow \Gamma_{w}^{\epsilon}$, we denote

$$
H_{\tau}(x)=\sum_{v \in \Gamma_{w}^{\epsilon}} a_{\tau(v)} \xi(x-v)
$$

Lemma 3.22. For any bijection $\tau: \Gamma_{w}^{\epsilon} \rightarrow \Gamma_{w}^{\epsilon}$, one has $\left\|H_{\tau}\right\|_{\mathcal{F}, \max }=\|H\|_{\mathcal{F}, \max }$.

Proof of Lemma 3.22. It is not hard to check that every bijection $\tau: \Gamma_{w}^{\epsilon} \rightarrow \Gamma_{w}^{\epsilon}$, can be written as a product of transpositions that interchange two neighboring points of $\Gamma_{w}^{\epsilon}$ (here, by neighboring points we mean $v^{\prime}, v^{\prime \prime} \in \Gamma_{w}^{\epsilon}$, such that $\left|v^{\prime}-v^{\prime \prime}\right|=4 \epsilon$ ). Therefore it is enough to prove the lemma for the case of such a transposition. 
Let $v^{\prime}=\left(z_{1}^{\prime}, \ldots, z_{n}^{\prime}\right), v^{\prime \prime}=\left(z_{1}^{\prime \prime}, \ldots, z_{n}^{\prime \prime}\right) \in \Gamma_{w}^{\epsilon}$ be a pair of neighboring points, where $z_{i}^{\prime}, z_{i}^{\prime \prime} \in \gamma_{i}$ for $i=1,2, \ldots, n$. There exists $1 \leqslant k \leqslant n$, such that $z_{i}^{\prime}=z_{i}^{\prime \prime}$ for $i \neq k$, and moreover $z_{k}^{\prime \prime}=z_{k}^{\prime} \pm 4 \epsilon$ or $z_{k}^{\prime \prime}=z_{k}^{\prime} \pm 4 \epsilon i$. The union of the neighboring squares $Q^{\prime}:=z_{k}^{\prime}+[-\epsilon, \epsilon]^{2}$, and $Q^{\prime \prime}:=z_{k}^{\prime \prime}+[-\epsilon, \epsilon]^{2}$ is a rectangle $S=Q^{\prime} \cup Q^{\prime \prime}$. Since the support $\operatorname{supp}\left(\xi_{k}\right) \subset(-\epsilon, \epsilon)^{2}$, there exists $0<\epsilon_{1}<\epsilon$, such that $\operatorname{supp}\left(\xi_{k}\right) \subset\left[-\epsilon_{1}, \epsilon_{1}\right]^{2}$. Looking on $Q_{1}^{\prime}=z_{k}^{\prime}+\left[-\epsilon_{1}, \epsilon_{1}\right]^{2}, Q_{1}^{\prime \prime}=z_{k}^{\prime \prime}+\left[-\epsilon_{1}, \epsilon_{1}\right]^{2} \subset \operatorname{int}(S)$, one can clearly move $Q_{1}^{\prime}$ to $Q_{2}^{\prime}$ and $Q_{2}^{\prime}$ to $Q_{1}^{\prime}$ simultaneously, using affine translations, such that at every moment the images of $Q_{1}^{\prime}, Q_{2}^{\prime}$ will not intersect, and are contained in $\operatorname{int}(S)$. Moreover, this can be done by a smooth Hamiltonian isotopy $\Phi_{K_{k}}^{t}$, supported in $S$, where $K_{k}\left(t, z_{k}\right):[0,1] \times W_{k} \rightarrow \mathbb{R}$ is the Hamiltonian that generates this isotopy, and such that we have $\operatorname{supp}\left(K_{k}(t, \cdot)\right) \subset \operatorname{int}(S)$ for all $t \in[0,1]$. For any $j \neq k$, $1 \leqslant j \leqslant n$ consider a smooth function $K_{j}\left(z_{j}\right): W_{j} \rightarrow \mathbb{R}$ such that $K_{j}\left(z_{j}\right)=1$ for $z_{j} \in z_{j}^{\prime}+[-\epsilon, \epsilon]^{2}$ and $K_{j}\left(z_{j}\right)=0$ for $z_{j} \in W_{j} \backslash\left(z_{j}^{\prime}+[-2 \epsilon, 2 \epsilon]^{2}\right)$. Now define a Hamiltonian $K:[0,1] \times W \rightarrow \mathbb{R}$ by

$$
K\left(t ; z_{1}, z_{2}, \ldots, z_{n}\right)=K_{k}\left(t, z_{k}\right) \prod_{\substack{1 \leqslant j \leqslant n \\ j \neq k}} K_{j}\left(z_{j}\right)
$$

Note that $K\left(t ; z_{1}, z_{2}, \ldots, z_{n}\right)=K_{k}\left(t, z_{k}\right)$ for

$$
z=\left(z_{1}, \ldots, z_{n}\right) \in U_{1}:=\prod_{j=1}^{k-1}\left(z_{j}^{\prime}+[-\epsilon, \epsilon]^{2}\right) \times S \times \prod_{j=k+1}^{n}\left(z_{j}^{\prime}+[-\epsilon, \epsilon]^{2}\right) .
$$

Moreover, $U_{1}$ is invariant under the flow $\Phi_{K}^{t}$, and

$$
\Phi_{K}^{t}\left(z_{1}, \ldots, z_{n}\right)=\left(z_{1}, \ldots, z_{k-1}, \Phi_{K_{k}}^{t}\left(z_{k}\right), z_{k+1}, \ldots, z_{n}\right),
$$

for any $z=\left(z_{1}, \ldots, z_{n}\right) \in U_{1}$. In particular, $\Phi_{K}^{1}(z)=z+v^{\prime \prime}-v^{\prime}$ for $z \in v^{\prime}+[-\epsilon, \epsilon]^{2 n}$, and $\Phi_{K}^{1}(z)=z+v^{\prime}-v^{\prime \prime}$ for $z \in v^{\prime \prime}+[-\epsilon, \epsilon]^{2 n}$. Furthermore, for

$$
U_{2}:=\prod_{j=1}^{k-1}\left(z_{j}^{\prime}+[-2 \epsilon, 2 \epsilon]^{2}\right) \times S \times \prod_{j=k+1}^{n}\left(z_{j}^{\prime}+[-2 \epsilon, 2 \epsilon]^{2}\right)
$$

we have that $\operatorname{supp}(K(t, \cdot)) \subset U_{2}$ for all $t \in[0,1]$. Therefore, since $\left(v+[-\epsilon, \epsilon]^{2 n}\right) \cap U_{2}=$ $\emptyset$ for all $v \in \Gamma_{w}^{\epsilon} \backslash\left\{v^{\prime}, v^{\prime \prime}\right\}$, we conclude that $\Phi_{K}^{1}(z)=z$ for $z \in v+[-\epsilon, \epsilon]^{2 n}$ for any $v \in \Gamma_{w}^{\epsilon} \backslash\left\{v^{\prime}, v^{\prime \prime}\right\}$. Hence if $\tau: \Gamma_{w}^{\epsilon} \rightarrow \Gamma_{w}^{\epsilon}$ is a transposition that interchanges $v^{\prime}$ with $v^{\prime \prime}$, we conclude that $H_{\tau}=\left(\Phi_{K}^{1}\right)^{*} H$. Therefore we conclude

$$
\left\|H_{\tau}\right\|_{\mathcal{F}, \max }=\|H\|_{\mathcal{F}, \max } .
$$

Proof of Lemma 3.21. Consider the decomposition $\Gamma_{w}^{\epsilon}=\prod_{i=1}^{n} \gamma_{i}$, and write each $\gamma_{i}$ explicitly as $\gamma_{i}=\left\{z_{i, 1}, \ldots, z_{i, N_{i}}\right\} \subset(-L, L)^{2}$. We order each set $\gamma_{i}$ by setting 
$z_{i, 1}<z_{i, 2}<\ldots<z_{i, N_{i}}$, for each $i$, and consider the lexicographic order $\prec$ on $\Gamma_{w}^{\epsilon}$ induced by these orders. We can arrange all the elements of $\Gamma_{w}^{\epsilon}$ by increasing order

$$
v_{1} \prec v_{2} \prec \ldots \prec v_{N},
$$

where $N=\prod_{i=1}^{n} N_{i}$. Take a bijection $\tau: \Gamma_{w}^{\epsilon} \rightarrow \Gamma_{w}^{\epsilon}$ such that

$$
a_{\tau\left(v^{\prime \prime}\right)} \leqslant a_{\tau\left(v^{\prime}\right)} \text { if and only if } v^{\prime} \preceq v^{\prime \prime}, \text { where } v^{\prime}, v^{\prime \prime} \in \Gamma_{w}^{\epsilon},
$$

and rewrite $H_{\tau}(x)=\sum_{v \in \Gamma_{w}^{\epsilon}} a_{\tau(v)} \xi(x-v)$ as

$$
H_{\tau}(x)=\sum_{j=1}^{N} b_{j} \xi\left(x-v_{j}\right), \text { and } b_{1} \leqslant b_{2} \leqslant \ldots \leqslant b_{N}
$$

By Lemma 3.22, one has $\left\|H_{\tau}\right\|_{\mathcal{F}, \max }=\|H\|_{\mathcal{F}, \max }$. Next, write

$$
H_{\tau}(x)=b_{N} K_{N}(x)+\sum_{j=1}^{N-1}\left(b_{j}-b_{j+1}\right) K_{j}(x),
$$

where $K_{j}(x)=\sum_{l=1}^{j} \xi\left(x-v_{j}\right)$. Also set $K_{0}(x)=0$.

$$
\begin{aligned}
\left\|H_{\tau}\right\|_{\mathcal{F}, \text { max }} & \leqslant\left|b_{N}\right|\left\|K_{N}(x)\right\|_{\mathcal{F}, \text { max }}+\sum_{j=1}^{N-1}\left|b_{j}-b_{j+1}\right|\left\|K_{j}\right\|_{\mathcal{F}, \text { max }} \\
& \leqslant\left|b_{N}\right|\left\|K_{N}(x)\right\|_{\mathcal{F}, \text { max }}+\sum_{j=0}^{N-1}\left(b_{j+1}-b_{j}\right) \max _{1 \leqslant j \leqslant N}\left\|K_{j}\right\|_{\mathcal{F}, \text { max }} \\
& =\left|b_{N}\right|\left\|K_{N}(x)\right\|_{\mathcal{F}, \text { max }}+\left(b_{N}-b_{1}\right) \max _{1 \leqslant j \leqslant N}\left\|K_{j}\right\|_{\mathcal{F}, \text { max }} \\
& \leqslant 3\left(\max _{v \in \Gamma_{w}^{\epsilon}}\left|a_{v}\right|\right) \max _{1 \leqslant j \leqslant N}\left\|K_{j}\right\|_{\mathcal{F}, \max }
\end{aligned}
$$

Next, consider some $K_{j}$, where $1 \leqslant j \leqslant N$. There exist a unique sequence

$$
j_{0}=0 \leqslant j_{1} \leqslant j_{2} \leqslant \ldots \leqslant j_{n-1} \leqslant j_{n}=j
$$

such that for any $1 \leqslant m \leqslant n$ we have $\prod_{l=m+1}^{n} N_{l} \mid j_{m}-j_{m-1}$, and we have

$$
k_{l}:=\frac{j_{m}-j_{m-1}}{\prod_{l=m+1}^{n} N_{l}}<N_{m}
$$

Here we mean $\prod_{l=n+1}^{n} N_{l}=1$. Take any $1 \leqslant m \leqslant n$. Then provided $j_{m-1}<j_{m}$, we can write

$$
\xi^{m}(z):=K_{j_{m}}-K_{j_{m-1}}=\prod_{l=1}^{n} \xi_{l}^{m}\left(z_{l}\right)
$$


where we have

$$
\begin{gathered}
\xi_{l}^{m}\left(z_{l}\right)=\xi_{l}\left(z_{l}-z_{l, k_{l}}\right), \text { for } l=1, \ldots, m-1, \\
\xi_{m}^{m}\left(z_{m}\right)=\sum_{i_{m}=1}^{k_{m}} \xi_{m}\left(z_{m}-z_{m, i_{m}}\right), \\
\xi_{l}^{m}\left(z_{l}\right)=\sum_{i_{l}=1}^{N_{l}} \xi_{l}\left(z_{l}-z_{l, i_{l}}\right), \text { for } l=m+1, \ldots, n .
\end{gathered}
$$

Moreover, for any $1 \leqslant m \leqslant n$ we have

$$
\left\|\xi^{m}\right\|_{\infty}=\prod_{l=1}^{n}\left\|\xi_{l}^{m}\right\|_{\infty}=\prod_{l=1}^{n}\left\|\xi_{l}\right\|_{\infty}=\|\xi\|_{\infty} .
$$

From this, and from Proposition 3.14 (i), we conclude that

$$
\left\|\xi^{m}\right\|_{\mathcal{F}, \max } \leqslant C\left\|\xi^{m}\right\|_{\infty}=C\|\xi\|_{\infty}
$$

for some $C=C(n)$. We have

$$
K_{j}=\sum_{m=1}^{n} \xi^{m}
$$

hence

$$
\left\|K_{j}\right\|_{\mathcal{F}, \max } \leqslant \sum_{m=1}^{n}\left\|\xi^{m}\right\|_{\mathcal{F}, \max } \leqslant n C\|\xi\|_{\infty}
$$

and this holds for any $1 \leqslant j \leqslant N$. Therefore we conclude

$$
\begin{gathered}
\|H\|_{\mathcal{F}, \text { max }}=\left\|H_{\tau}\right\|_{\mathcal{F}, \max } \leqslant 3\left(\max _{v \in \Gamma_{w}^{\epsilon}}\left|a_{v}\right|\right) \max _{1 \leqslant j \leqslant N}\left\|K_{j}\right\|_{\mathcal{F}, \max } \\
\leqslant 3 n C\left(\max _{v \in \Gamma_{w}^{\epsilon}}\left|a_{v}\right|\right)\|\xi\|_{\infty}=3 n C\|H\|_{\infty} .
\end{gathered}
$$

The proof of the lemma is now complete.

\section{Proof of Theorem 1.3}

The proof of Theorem 1.3 follows from Theorem 3.4 by a standard partition of unity argument. For the sake of completeness, we provide the details below.

As explained in Section 2, it is enough to prove Theorem 1.3 for $\operatorname{Ham}(M, \omega)$ invariant pseudo norms on $C^{\infty}(M)$. Indeed, any $\operatorname{Ham}(M, \omega)$-invariant pseudo norm $\|\cdot\|$ on $\mathcal{A}$ that is continuous in the $C^{\infty}$-topology, can be naturally extended to a 
$\operatorname{Ham}(M, \omega)$-invariant pseudo-norm $\|\cdot\|^{\prime}$ on $C^{\infty}(M)$, which is again continuous in the $C^{\infty}$-topology, by setting

$$
\|f\|^{\prime}=\left\|f-M_{f}\right\|, \text { where } M_{f}=\frac{1}{\operatorname{Vol}(M)} \int_{M} f \omega^{n}
$$

Consider a Darboux chart $i: U \hookrightarrow M$, where $U \subset\left(\mathbb{R}^{2 n}, \omega_{s t d}\right)$ is an open set. Without loss of generality we assume that the origin of $\mathbb{R}^{2 n}$ lies inside $U$. Choose some $L>0$, such that $W=(-L, L)^{2 n} \subset U$. Since $i(W) \subset M$, we have a natural embedding $C_{c}^{\infty}(i(W)) \hookrightarrow C^{\infty}(M)$, and therefore any $\operatorname{Ham}(M, \omega)$-invariant pseudo norm $\|\cdot\|$ on $C^{\infty}(M)$ restricts to $C_{c}^{\infty}(i(W))$. From Lemma 3.3 and Theorem 3.4, we conclude that (when the norm is continuous in the $C^{\infty}$-topology) there exists a constant $C>0$ such that

$$
\|f\| \leqslant C\|f\|_{\infty}, \text { for every function } f \in C_{c}^{\infty}(i(W))
$$

Next, for any point $x \in M$ there exists an open neighborhood $V_{x} \subset M$, and a smooth Hamiltonian diffeomorphism $\Phi_{x} \in \operatorname{Ham}(M, \omega)$, such that $\Phi_{x}\left(V_{x}\right) \subset W$. Consider the open covering $\bigcup_{x \in M} V_{x}=M$. The compactness of $M$ allows us to pass to a finite subcover $\bigcup_{i=1}^{N} V_{x_{i}}=M$. Moreover, one can find a partition of unity $\left\{\rho_{1}, \rho_{2}, \ldots, \rho_{N}\right\}$, such that for every $i=1,2, \ldots, N, \rho_{i}: M \rightarrow \mathbb{R}$ is a smooth positive function supported in $V_{x_{i}}$, and

$$
\rho_{1}+\rho_{2}+\ldots+\rho_{N}=\mathbb{1}_{M}
$$

Finally, let $f \in C^{\infty}(M)$, and consider the decomposition

$$
f=\rho_{1} f+\rho_{2} f+\ldots+\rho_{N} f
$$

Since $\|\cdot\|$ is a $\operatorname{Ham}(M, \omega)$-invariant norm, it follows that

$$
\|f\| \leqslant \sum_{i=1}^{N}\left\|\rho_{i} f\right\|=\sum_{i=1}^{N}\left\|\left(\Phi_{x_{i}}^{-1}\right)^{*}\left(\rho_{i} f\right)\right\|
$$

Moreover, it follows from the above that $\operatorname{supp}\left(\left(\Phi_{x_{i}}^{-1}\right)^{*}\left(\rho_{i} f\right)\right) \subset W$, and hence

$$
\left\|\left(\Phi_{x_{i}}^{-1}\right)^{*}\left(\rho_{i} f\right)\right\| \leqslant C\left\|\left(\Phi_{x_{i}}^{-1}\right)^{*}\left(\rho_{i} f\right)\right\|_{\infty}=C\left\|\rho_{i} f\right\|_{\infty} \leqslant C\|f\|_{\infty} .
$$

Therefore we conclude that

$$
\|f\| \leqslant C^{\prime}\|f\|_{\infty}
$$

where $C^{\prime}=N C$. The proof of the theorem is now complete.

\section{Appendix}

Here we prove the claim mentioned in Remark 1.1. More precisely: 
Proposition 5.1. Let $M$ be a closed symplectic manifold, and let $\|\cdot\|$ be a norm on the Lie algebra $\mathcal{A}$ of $\operatorname{Ham}(M, \omega)$. Then, smooth paths $[0,1] \rightarrow \operatorname{Ham}(M, \omega)$ have finite length if and only if the norm $\|\cdot\|$ is continuous in the $C^{\infty}$-topology.

Proof. The "if" part of the statement is clear. Let us show the "only if" part. Throughout, we equip $M$ with a Riemmanian metric, and denote $\|\cdot\|_{\infty}=\|\cdot\|_{C^{0}} \leqslant$ $\|\cdot\|_{C^{1}} \leqslant\|\cdot\|_{C^{2}} \leqslant \ldots$ the corresponding $C^{0}, C^{1}, C^{2}, \ldots$-norms on $C^{\infty}(M)$.

Let $\|\cdot\|$ be an invariant pseudo-norm on $C^{\infty}(M)$ which is not continuous in the $C^{\infty}$-topology. Consider two sequences $\left\{a_{k}\right\},\left\{b_{k}\right\}$ in the interval $[0,1]$, such that

$$
0<a_{1}<b_{1}<a_{2}<b_{2}<\ldots<1
$$

Next, let $c:[0,1] \rightarrow[0,1]$ be a smooth function such that $c(t)=0$ for $t \in\left[0, \frac{1}{4}\right] \cup\left[\frac{3}{4}, 1\right]$, and $c(t)=1$ for $t \in\left[\frac{1}{3}, \frac{2}{3}\right]$. For a sequence of smooth functions $H_{k}: M \rightarrow \mathbb{R}$, we define a function $H: M \times[0,1] \rightarrow \mathbb{R}$ in the following way:

$$
H(x, t)= \begin{cases}0 & \text { for } t \in\left[0, a_{1}\right] \cup\left[b_{1}, a_{2}\right] \cup\left[b_{2}, a_{3}\right] \cup \ldots, \\ c\left(\frac{t-a_{k}}{b_{k}-a_{k}}\right) H_{k}(x) & \text { for } t \in\left[a_{k}, b_{k}\right] \\ 0 & \text { for } t=1 .\end{cases}
$$

Note that $H$ is smooth on $M \times[0,1)$. We next show that for a suitable choice of a sequence $H_{k} \in C^{\infty}(M)$, one has $H(x, t) \in C^{\infty}(M \times[0,1])$, and moreover

$$
\int_{0}^{1}\|H(\cdot, t)\| d t=+\infty
$$

Thus, the Hamiltonian flow of $H$ has infinite length with respect to the Finsler metric $d_{\|\cdot\| \cdot}$. Indeed, note that

$$
\int_{0}^{1}\|H(\cdot, t)\| d t=\sum_{k=1}^{\infty}\left(b_{k}-a_{k}\right)\left(\int_{0}^{1}|c(t)| d t\right)\left\|H_{k}\right\| \geqslant \frac{1}{3} \sum_{k=1}^{\infty}\left(b_{k}-a_{k}\right)\left\|H_{k}\right\| .
$$

Hence, for the estimate (5.2), it is enough to choose $H_{k}$ such that $\left\|H_{k}\right\| \geqslant \frac{1}{b_{k}-a_{k}}$. Moreover, to ensure that $H(x, t)$ is smooth in $M \times[0,1]$, it is enough to have

$$
\lim _{t \rightarrow 1}\left\|\frac{\partial^{j}}{\partial t^{j}} H(t, \cdot)\right\|_{C^{m}}=0, \text { for any } j, m \geqslant 0
$$

More precisely, assume that $t \in\left(a_{k}, b_{k}\right)$. Note that in that case

$$
\left\|\frac{\partial^{j}}{\partial t^{j}} H(t, \cdot)\right\|_{C^{m}}=\left(\frac{1}{b_{k}-a_{k}}\right)^{j}\left|c^{(j)}\left(\frac{t-a_{k}}{b_{k}-a_{k}}\right)\right|\left\|H_{k}\right\|_{C^{m}} \leqslant\left(\frac{1}{b_{k}-a_{k}}\right)^{j}\|c\|_{C^{j}}\left\|H_{k}\right\|_{C^{m}} .
$$

Therefore, to show (5.3) it is enough to choose $H_{k}$ such that

$$
\lim _{k \rightarrow \infty}\left(\frac{1}{b_{k}-a_{k}}\right)^{j}\left\|H_{k}\right\|_{C^{m}}=0, \text { for any } j, m \geqslant 0
$$


In particular, any sequence $H_{k} \in C^{\infty}(M)$, that for every $k \geqslant 1$ satisfy

$$
\left\{\begin{array}{l}
\left\|H_{k}\right\| \geqslant \frac{1}{b_{k}-a_{k}}, \\
\left\|H_{k}\right\|_{C^{k}} \leqslant\left(b_{k}-a_{k}\right)^{k},
\end{array}\right.
$$

would give rise (via definition (5.1) ) to a smooth function $H: M \times[0,1] \rightarrow \mathbb{R}$, such that $\int_{0}^{1}\|H(\cdot, t)\| d t=+\infty$.

Since the norm $\|\cdot\|$ is assumed to be non-continuous in the $C^{\infty}$-topology, one can always find a sequence $\left\{H_{k}\right\}$ which satisfy (5.4).

\section{References}

[1] Arnold, V. I. Sur la géométrie différentielle des groupes de Lie de dimension infinie et ses applications à l'hydrodynamique des fluides parfaits, (French) Ann. Inst. Fourier (Grenoble) 161966 fasc. 1 319-361.

[2] Arnold, V. I., Khesin, B. A. Topological methods in hydrodynamics, Applied Mathematical Sciences, 125. Springer-Verlag, New York, 1998.

[3] Banyaga, A. Sur la structure du groupe des difféomorphisms qui préservent une forme symplectique. Comment. Math. Helv. 53 (1978), no.2, 174-227.

[4] Eliashberg, Y. Symplectic topology in the nineties, Symplectic geometry. Differential Geom. Appl. 9 (1998), no. 1-2, 59-88.

[5] Eliashberg, Y., Polterovich, L. Bi-invariant metrics on the group of Hamiltonian diffeomorphisms, Internat. J. Math. 4 (1993), 727-738.

[6] Hofer, H. On the topological properties of symplectic maps. Proceedings of the Royal Society of Edinburgh, 115 (1990), 25-38.

[7] Hofer, H., Zehnder, E. Symplectic Invariants and Hamiltonian Dynamics, Birkhäuser Advanced Texts, Birkhäuser, Basel (1994).

[8] Khesin, B. A., Wendt, R. The geometry of infinite-dimensional groups, Ergebnisse der Mathematik, vol. 51. Springer, New York (2008).

[9] Körner, T. W. Fourier analysis, Cambridge University Press, Cambridge, 1988.

[10] Lalonde, F., McDuff, D. The geometry of symplectic energy, Ann. of Math 141 (1995), 349-371. Math 141 (1995), 349-371. 
[11] McDuff, D., Salamon, D. Introduction to Symplectic Topology, 2nd edition, Oxford University Press (1998).

[12] Oh, Y.-G. Spectral invariants, analysis of the Floer moduli space, and geometry of the Hamiltonian diffeomorphism group, Duke Math. J. 130 (2005), 199-295.

[13] Ostrover, Y., Wagner, R. On the extremality of Hofer's metric on the group of Hamiltonian diffeomorphisms, Inter. Math. Res. Notices 35, 2123-2142 (2005).

[14] Polterovich, L. Symplectic displacement energy for Lagrangian submanifolds, Ergodic Theory and Dynamical Systems, 13 (1993), 357-67.

[15] Polterovich, L. The geometry of the group of symplectic diffeomorphisms, Lectures in Mathematics ETH Zürich. Birkhäuser Verlag, Basel, 2001.

[16] F. Schlenk, Embedding problems in symplectic geometry, De Gruyter Expositions in Mathematics, 40, Walter de Gruyter Verlag, Berlin, 2005.

[17] Schwarz, M. On the action spectrum for closed symplectically aspherical manifolds, Pacific J. Math. 193 (2000), 1046-1095.

[18] Viterbo, C. Symplectic topology as the geometry of generating functions, Math. Ann. 292 (1992) 685-710.

Lev Buhovsky

The Mathematical Sciences Research Institute, Berkeley, CA 94720-5070

e-mail: levbuh@gmail.com

Yaron Ostrover

School of Mathematics, Institute for Advanced Study, Princeton NJ 08540

e-mail: ostrover@math.ias.edu 